\title{
A brief review of the biological potential of indole derivatives
}

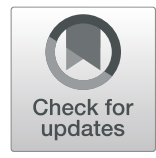

\author{
Sunil Kumar ${ }^{*}$ and Ritika
}

\begin{abstract}
Background: Various bioactive aromatic compounds containing the indole nucleus showed clinical and biological applications. Indole scaffold has been found in many of the important synthetic drug molecules which gave a valuable idea for treatment and binds with high affinity to the multiple receptors helpful in developing new useful derivatives.

Main text: Indole derivatives possess various biological activities, i.e., antiviral, anti-inflammatory, anticancer, antiHIV, antioxidant, antimicrobial, antitubercular, antidiabetic, antimalarial, anticholinesterase activities, etc. which created interest among researchers to synthesize a variety of indole derivatives.
\end{abstract}

Conclusion: From the literature, it is revealed that indole derivatives have diverse biological activities and also have an immeasurable potential to be explored for newer therapeutic possibilities.

Keywords: Indole, Antiviral, Anti-inflammatory, Anticancer, Anti-HIV, Antioxidant, Antimicrobial, Antitubercular, Antidiabetic, Antimalarial, Anticholinesterase activities

\section{Background}

Indole is also known as benzopyrrole which contains benzenoid nucleus and has $10 \pi$-electrons (two from lone pair on nitrogen and double bonds provide eight electrons) which makes them aromatic in nature. Similar to the benzene ring, electrophilic substitution occurs readily on indole due to excessive $\pi$-electrons delocalization [1]. Indole is an important heterocyclic system that provides the skeleton to lysergic acid diethylamide (LSD), strychnine, and alkaloid obtained from plants. Physically, they are crystalline colorless in nature with specific odors. The addition of the indole nucleus to medicinal compounds that is biologically active pharmacophore made it an important heterocyclic compound having broad-spectrum biological activities [2]. Due to this, researchers took interest to synthesize various scaffolds of indole for screening different pharmacological activities. Various natural

\footnotetext{
*Correspondence: sunilkumar188@gmail.com
}

Drug Discovery and Research Lab, Department of Pharmaceutical Sciences, Guru Jambheshwar University of Science \& Technology, Hisar, Haryana, India compounds contain indole as parent nucleus for example tryptophan. Indole-3-acetic acid is a plant hormone produced by the degradation of tryptophan in higher plants. Derivatives of indole are of wide interest because of their diverse biological and clinical applications. Here, we have tried to summarize the important pharmacological activity of indole derivatives [3].

\section{Main text \\ Biological activities of indole Antiviral activity}

6-Amino-4-substitutedalkyl- $1 H$-indole-2-substitutedcarboxylate derivatives were prepared and reported as antiviral agents by Xue et al. In all tested compounds, compound methyl 6-amino-4-isobutoxy- $1 \mathrm{H}$ indole-2-carboxylate (1) showed inhibitory activity against influenza $\mathrm{A}$ with $\mathrm{IC}_{50}=7.53 \mu \mathrm{mol} / \mathrm{L}$ and the highest selectivity index (SI) value 17.1 to CoxB3 virus [4].

\section{Springer Open}

Ñ The Author(s). 2020 Open Access This article is licensed under a Creative Commons Attribution 4.0 International License, which permits use, sharing, adaptation, distribution and reproduction in any medium or format, as long as you give appropriate credit to the original author(s) and the source, provide a link to the Creative Commons licence, and indicate if changes were made. The images or other third party material in this article are included in the article"s Creative Commons licence, unless indicated otherwise in a credit line to the material. If material is not included in the article"s Creative Commons licence and your intended use is not permitted by statutory regulation or exceeds the permitted use, you will need to obtain permission directly from the copyright holder. To view a copy of this licence, visit http://creativecommons.org/licenses/by/4.0/. 
<smiles>COC(=O)c1cc2c(OC)cc(N)cc2[nH]1</smiles>

(1)

4-Alkyl-1-(5-fluoro-3-phenyl-1 $H$-indole-2-carbonyl)thiosemicarbazide derivatives of indole were prepared and investigated in vitro for antiviral activity in a broad range of ribonucleic acid (RNA) and deoxyribonucleic acid (DNA) viruses by Cihan-Üstündag et al. Compounds 1-(5-fluoro-3-phenyl-1H-indole-2carbonyl)-4-methylthiosemicarbazide (2), 4-ethyl-1-(5fluoro-3-phenyl- $1 H$-indole-2-carbonyl)thiosemicarbazide (3), 1-(5-fluoro-3-phenyl-1 $H$-indole-2-carbonyl)-4propylthiosemicarbazide (4), and 4-butyl-1-(5-fluoro3-phenyl-1 $H$-indole-2-carbonyl)thiosemicarbazide (5) are potent antiviral agents with $\mathrm{IC}_{50}$ values ranging from 0.4 to $2.1 \mu \mathrm{g} / \mathrm{mL}$ against Coxsackie B4 virus [5].

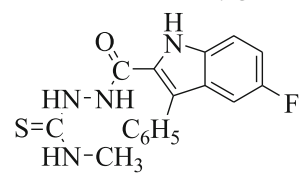

(2)

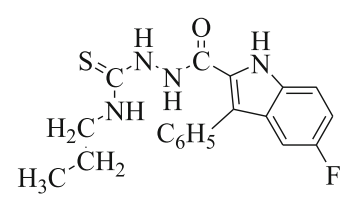

(4)

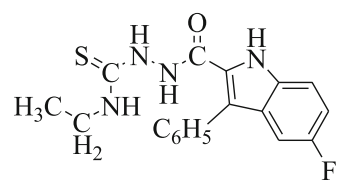

(3)

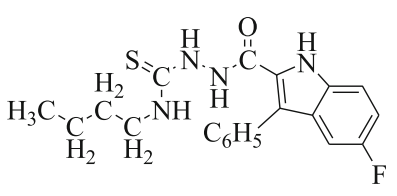

(5)
Ethyl $1 H$-indole-3-carboxylates also showed antiviral activity in Huh-7.5 cells explained by Sellitto et al. Compound 4-((3-(ethoxycarbonyl)-1-methyl-5(pyrrolidin-1-ylmethyl)-1H-indol-2-yl)methyl)benzenesulfinate (6) was the most active compound at low concentration against hepatitis $\mathrm{C}$ virus (HCV) [6].

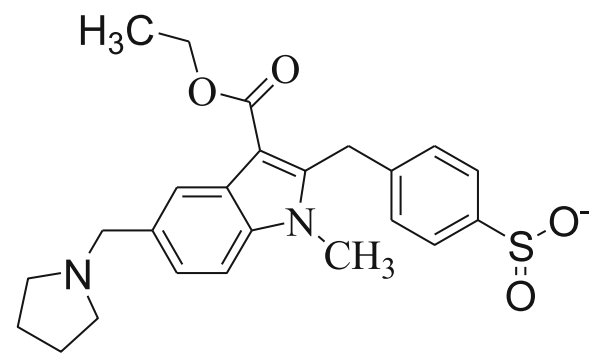

(6)
Giampieri et al. elaborated reaction of indoles and 2naphthols through Mannich bases and tested against different viruses and compound methyl 1-( $(1 H$-indol-3yl)methyl)-2-naphthoate (7) showed significant activity against Yellow Fever Virus (YFV), Bovine viral diarrhea virus (BVDV), Human immunodeficiency virus-1 (HIV1 ), and Respiratory syncytial virus (RSV) [7].<smiles>CC(=O)c1ccc2ccccc2c1Cc1c[nH]c2ccccc12</smiles>

(7)

Pyrimidine-derived indole ribonucleosides $(2 S, 3 R, 4 S$, $5 S$ )-2-(6-chloro-4-(furan-2-yl)-9H-pyrimido[4,5-

b]indol-9-yl)-5-(hydroxymethyl)-tetrahydrofuran-3,4-diols were synthesized and tested for in vitro antiproliferative (HL-60 cervical carcinoma HeLaS3, Tlymphoblastic leukemia human cell line CCRF-CEM andpromyelocyticleukemia) and antiviral activity (Dengue virus and anti-hepatitis $C$ virus) by Tichy et al. Compound (2S,3R,4S,5S)-2-(6-chloro-4-(furan-2-yl)9H-pyrimido[4,5-b]indol-9-yl)-5-(hydroxymethyl)-tetrahydrofuran-3,4-diol (8) exhibited the notable cytotoxicity in HepG2 cells and THP-1 with $\mathrm{IC}_{50}$ of 0.175 and $1.565 \mu \mathrm{M}$ [8].<smiles>OC[C@H]1O[C@@H](n2c3ccc(Cl)cc3c3c(-c4ccco4)ncnc32)C(O)C1O</smiles>

(8)

5-Nitro-3-[(5-nonsubstituted/methyl-4-thiazolidinone-2ylidene) hydrazono]-1H-2-indolinones were prepared and tested for antiviral activities by Terzioğlu et al. Compounds (Z)-4-allyl-1-(1-(morpholinomethyl)-5-nitro-2-oxoindolin3-ylidene)thiosemicarbazide (9), (3Z,3E)-3-(2-(3-ethyl-4oxothiazolidin-2-ylidene)hydrazono)-5-nitroindolin-2-one (10), (3Z,3E)-5-nitro-3-(2-(4-oxo-3-phenylthiazolidin-2-ylidene)hydrazono)indolin-2-one (11), (3Z,3E)-3-(2-(3-(4-bromophenyl)-5-methyl-4-oxothiazolidin-2-ylidene)hydrazono)-5-nitroindolin-2-one (12) and (3Z,3E)-3-(2-(3-(4chlorophenyl)-5-methyl-4-oxothiazolidin-2-ylidene)hydrazono)-5-nitroindolin-2-one (13) prevented the development of bovine viral diarrhea virus in cells [9]. 


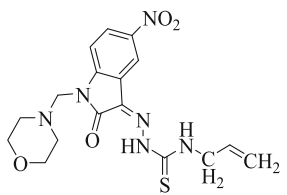

(9)

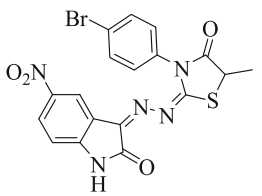

(12)

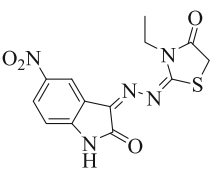

(10)

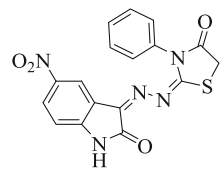

(11)

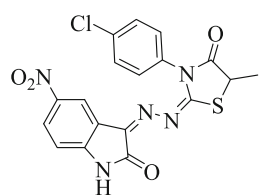

(13)
7-Ethoxy-1-methyl-4, 9-dihydro-3H-pyrido [3, 4-b] indole derivatives were reported as anti-Herpes Simplex virus-1(HSV-1) compounds by El-sawy et al. and derivatives ethyl 2-(3-(5-(4-nitrophenyl)-1-phenyl-4,5-dihydro$1 H$-pyrazol-3-yl)-1H-indol-1-yl)acetate (14), ethyl 2-(3(6-(4-nitrophenyl)-2-oxo-1,2-dihydropyrimidin-4-yl)- $1 H$ indol-1-yl)acetate (15), ethyl 2-(3-(6-(4-nitrophenyl)-2thioxo-1,2-dihydropyrimidin-4-yl)-1 $H$-indol-1-yl)acetate (16) and ethyl 2-(3-(6-(4-chlorophenyl)-2-imino-1,2dihydropyrimidin-4-yl)- $1 H$-indol-1-yl)acetate (17) possessed considerable antiviral activity with $\mathrm{IC}_{50}$ ranged between 5 and $6 \mu \mathrm{g} / \mathrm{ml}$ and substantial therapeutic indices (TI) of 80 and 83 were recorded [10].<smiles>CCOC(=O)Cn1cc(C2=NN(c3ccccc3)C(c3ccc([N+](=O)[O-])cc3)C2)c2ccccc21</smiles>

(14)<smiles>CCOC(=O)Cn1cc(-c2cc(-c3ccc([N+](=O)[O-])cc3)[nH]c(=S)n2)c2ccccc21</smiles>

(16)<smiles>CCOC(=O)Cn1cc(-c2cc(-c3ccc([N+](=O)[O-])cc3)[nH]c(=O)n2)c2ccccc21</smiles>

(15)<smiles>CCOC(=O)Cn1cc(-c2cc(-c3ccc(Cl)cc3)[nH]c(=N)n2)c2ccccc21</smiles>

(17)
1,3-Thiazole and 1,2,4-triazolo[3,4-b]1,3,4-thiadiazine containing indole nucleus derivatives were prepared and checked for their antiviral activity against HSV-1(herpes simplex type1) by Abdel-gawad et al. Compounds 5-(1H-indol-3-yl)-1-phenyl- $N$ - $(4,5,6,7$ tetrachloro-1,3-dioxoisoindolin-2-yl)-1H-pyrazole-3carboxamide (18) and 2-(2-((E)-1-(1H-indol-3yl)ethylidene)hydrazinyl)-5-((E)-(4-chlorophenyl)diazenyl)-4-methylthiazole (19) showed best activity against HSV-1 [11].

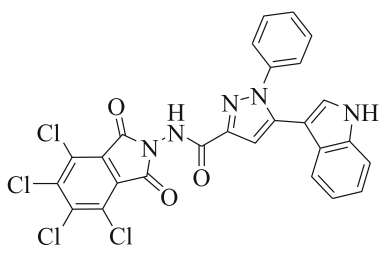

(18)

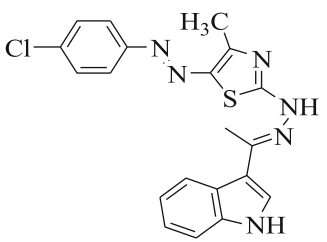

(19)
Indole-based spirothiazolidinones also have antiviral activity as discussed by Cihan-Üstündağ et al. Compounds presented inhibitory action in Vero cells against yellow fever and Punta Toro virus. The range of $\mathrm{IC}_{50}$ values was $1.9-12 \mu \mathrm{M}$. Compound 5-chloro- $N-((2 S, 5 S$, 8R)-2,8-dimethyl-3-oxo-1-thia-4-azaspiro[4.5]decan-4yl)-3-phenyl- $1 H$-indole-2-carboxamide (20) was the most active [12].<smiles>CC1CCC2(CC1)SC(C)C(=O)N2NC(=O)c1[nH]c2ccc(Cl)cc2c1-c1ccccc1</smiles>

\section{(20)}

\section{Anti-inflammatory and analgesic activities}

Indole-based chalcone derivatives reported as COX-1 and COX-2 inhibitor by Ozdemir et al. Compound 3-(5Bromo-1H-indol-3-yl)-1-(4-cyanophenyl)prop-2-en-1one (21) and compound 3-(5-methoxy-1H-indol-3-yl)-1(4-(methylsulfonyl)phenyl)prop-2-en-1-one (22) were found to demonstrate a significant activity [13].<smiles>N#Cc1ccc(C(=O)/C=C/c2c[nH]c3ccc(Br)cc23)cc1</smiles>

(21)<smiles>COc1ccc(C(=O)/C=C/c2c[nH]c3ccc(OC)cc23)cc1</smiles>

(22)
Sarva et al. carried out solvent-free reaction in microwave between indole and substituted aldehydes. The product, bis(indolyl)methane is bioactive. The anti-inflammatory activity was shown by most of the compounds but compounds 3,3'-([1,1'-biphenyl $]-4$ ylmethylene)bis( $1 H$-indole) $\quad(23), \quad 3,3 '-((1 H$-imidazol-2yl)methylene)bis( $1 H$-indole) (24), 3,3'-((5-methylpyridin-2-yl)methylene)bis(1H-indole) (25) and 3,3'-(thiophen-2-ylmethylene)bis( $1 H$-indole) (26) were the most potent [14]. 


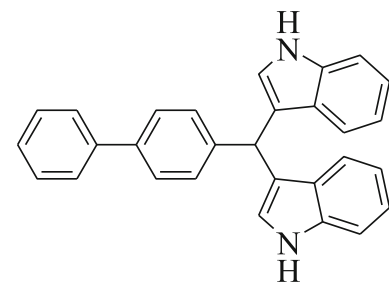

(23)<smiles>Cc1ccc(C(c2c[nH]c3ccccc23)c2c[nH]c3ccccc23)nc1</smiles>

(25)<smiles>c1ccc2c(C(c3ncc[nH]3)c3c[nH]c4ccccc34)c[nH]c2c1</smiles>

(24)<smiles>c1csc(C(c2c[nH]c3ccccc23)c2c[nH]c3ccccc23)c1</smiles>

(26)
Anti-inflammatory activities of chalcones of indole were elaborated by Rani et al. against carrageenaninduced edema in albino rats. The most effective compound of this series was found to be 4-(3- $(1 H$ - indol-3yl)-4, 5-dihydro-1H-pyrazol-5-yl) phenol (27) [15].<smiles>Oc1ccc(C=Cc2c[nH]c3ccccc23)cc1</smiles>

(27)

Indole containing isoxazole derivatives were reported by Pedada et al. as $\mathrm{SPLA}_{2}$ inhibitory agents. Compound $N$-((3-(4-fluoro-3-(trifluoromethyl) phenyl) isoxazol-5-yl) methyl) (5-methyl-1H-indol-3-yl) methanamine hydrochloride (28) showed significant $\mathrm{SPLA}_{2}$ inhibition activity that is comparable or more to ursolic acid (positive control) [16].<smiles>Cc1ccc2[nH]cc(CNCc3cc(-c4ccc(F)c(C(F)(F)F)c4)no3)c2c1</smiles>

(28)

Reactive oxygen species (ROS) generation and nitric oxide release induced through lipopolysaccharide were inhibited free radicals HMPH (1-[(1H-indol-3-yl)methylene]-2-phenylhydrazine scavenged) in RAW cells without any cytotoxicity explained by Misra et al. In all tested compounds, compound (E)-1-((1H-indol-3-yl)methylene)2-phenylhydrazine (29) showed significant activity [17].<smiles>C(=N/Nc1ccccc1)\c1c[nH]c2ccccc12</smiles>

(29)

3-(2'-Substituted indolidene aminothiazol-4'-yl)-2-(4chlorophenyl) indoles derivatives were reported as analgesic and anti-inflammatory agents by Singh et al. Compound (E)-4-(2-(4-chlorophenyl)-1H-indol-3-yl)- $N$-((2methyl- $1 H$-indol-3-yl)methylene)thiazol-2-amine (30) showed the better result as anti-inflammatory and analgesic agent [18].<smiles>Cc1[nH]c2ccccc2c1C=Nc1nc(-c2c(-c3ccc(Cl)cc3)[nH]c3ccccc23)cs1</smiles>

(30)

(3-Ethyl-5-(methylsulfonyl)-2-phenyl-1H-indol-1-yl) substitutedphenyl methanones were prepared and tested for their anti-inflammatory activity against COX enzymes. For in vitro studies, all of the tested compounds, especially compounds incorporating $\mathrm{SO}_{2} \mathrm{Me}$ moiety as a COX-2 pharmacophoric feature, showed preferential inhibitory activity against COX2 over COX-1(SI $=4.02$ to 65.71$)$ compared with indomethacin $(\mathrm{SI}=0.079)$. Whereas in vivo anti-inflammatory activity was good for the compound having $\mathrm{SO}_{2} \mathrm{Me}$ moiety and compounds (3-ethyl-5-(methylsulfonyl)-2-phenyl- $1 H$-indol1-yl)(4-fluorophenyl)methanone (31) and (4-chlorophenyl)(3ethyl-5-(methylsulfonyl)-2-phenyl- $1 H$-indol-1-yl)methanone (32) were more potent than indomethacin. The presence of a carbonyl moiety as a spacer instead of methylene resulted in an increase in the anti-inflammatory activity [19]

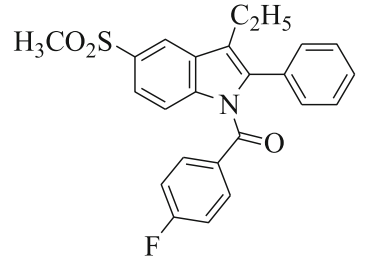

(31)<smiles>CCc1c(-c2ccccc2)n(C(=O)c2ccc(Cl)cc2)c2ccc(OC)cc12</smiles>

(32) 
3-Methyl-2-phenyl-1-substituted-indole derivatives were synthesized and investigated for anti-inflammatory (in vitro and in vivo) and analgesic activities by Abdellatif et al. Derivatives (3-methyl-5-(methylsulfonyl)-2phenyl-1H-indol-1-yl)(phenyl)methanone (33), (4-chlorophenyl)(3-methyl-5-(methylsulfonyl)-2-phenyl-1Hindol-1-yl)methanone (34), and 1-benzyl-3-methyl-5(methylsulfonyl)-2-phenyl-1 $\mathrm{H}$-indole (35) showed the highest anti-inflammatory (in vitro and in vivo) and analgesic activities. The results of molecular docking studies were in agreement with that obtained from the in vitro COX inhibition assays [20].

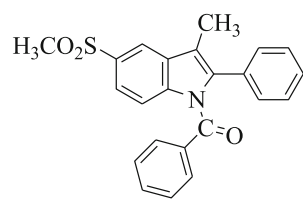

(33)

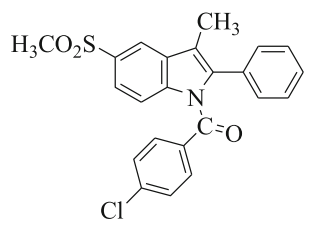

(34)

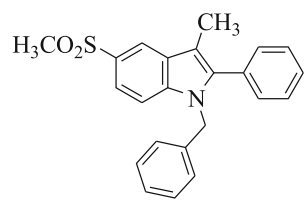

(35)

3-(2-Aminopyrimidin-4-yl) indoles were prepared and investigated for their ulcerogenic, anti-inflammatory, and analgesic activities by Chavan et al. All the synthesized compounds showed alike results with indomethacin. Among the tested compounds, compounds 4-(2amino-6-(2-(4-chlorophenyl)- $1 H$-indol-3-yl)pyrimidin-4yl)phenol (36) and 4-(4-aminophenyl)-6-(2-(4-chlorophenyl)-1 $H$-indol-3-yl)pyrimidin-2-amine (37) showed 87.4 and $88.2 \%$ inflammation inhibition using paw edema, 78.5 and $76.6 \%$ inhibition of acetic acid-induced writhings [21].

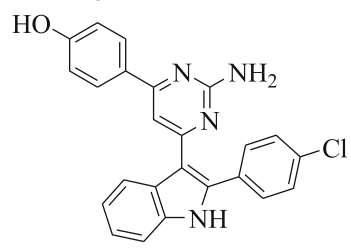

(36)

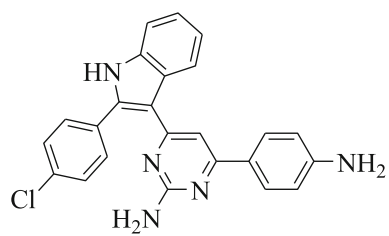

(37)
Some new derivatives of 3-(2'-carboxy-5'-mehoxyindol-3'-yl)-5-(substituted phenyl)-2-isoxazolines have been prepared with less CVS and CNS activities but good anti-inflammatory activity by Prajapati et al. All the compounds were investigated for anti-inflammatory activity and compound 5methoxy-3-(3-phenylacryloyl)- $1 \mathrm{H}$-indole-2-carboxylic acid (38) showed maximum activity $(64.20 \%)$ at $50 \mathrm{mg} / \mathrm{kg}$ [22].<smiles>COc1ccc2[nH]c(C(=O)O)c(C(=O)C=Cc3ccccc3)c2c1</smiles>

\section{(38)}

1, 8-Dihydro-1-aryl-8-alkyl pyrazolo(3,4-b)indoles were synthesized by Mandour et al. that showed remarkable anticonvulsant, analgesic, and anti-inflammatory activities in comparison to diazepam, flufenamic acid, and indomethacin as positive controls. Among the tested compounds, the most potent compounds were found to be (6-chlorocyclohexa-2,4-dienyl)(1-(2,4,6-trichlorophenyl)pyrazolo[3,4-b]indol-8(1H)-yl)methanone (39) and (6-chlorocyclohexa-2,4-dienyl)(1-phenylpyrazolo[3,4b]indol-8 $(1 \mathrm{H})$-yl)methanone (40) with percentage inflammation inhibition 85 and $79 \%\left(100 \mathrm{mg} \mathrm{kg}^{-1}\right)$ and 70 and $64 \%\left(50 \mathrm{mg} \mathrm{kg}^{-1}\right)$ respectively [23].<smiles>O=C(C1C=CC=CC1Cl)n1c2ccccc2c2cnn(-c3c(Cl)cc(Cl)cc3Cl)c21</smiles>

(39)<smiles>O=C(C1C=CC=CC1Cl)n1c2ccccc2c2cnn(-c3ccccc3)c21</smiles>

(40)
2-(5-Methoxy-2-methyl- $1 H$-indol-3- $y l)-N^{\prime}-[(E)$ (substituted phenyl)methylidene] acetohydrazide derivatives were reported by Bhat et al. and investigated for cyclooxygenase expression, lipid peroxidation, ulcerogenic, analgesic, and anti-inflammatory activities. Compound (E)- $N^{\prime}$-(3-nitrobenzylidene)-2-(5-methoxy-2-methyl-1Hindol-3-yl) acetohydrazide (41) was active as analgesic and anti-inflammatory agents [24].<smiles>COc1ccc2[nH]c(C)c(CC(=O)N/N=C/c3cccc([N+](=O)[O-])c3)c2c1</smiles>

(41)

Benzothiazole containing benzene sulphonamide and carboxamide were prepared by Ugwu et al. and evaluated for their in vivo anti-inflammatory, analgesic, and ulcerogenic activities. Amongst the derivatives, compounds (S)- $N$-(1-(benzo[d]thiazol-2-ylamino)-3-(1Hindol-2-yl)-1-oxopropan-2-yl)- $N$-(4- 
nitrophenylsulfonyl)benzamide (42) and (S)-N-(benzo[d]thiazol-2-yl)-1-(4-nitrophenylsulfonyl)pyrrolidine-2carboxamide (43) showed anti-inflammatory and analgesic activities along with ulcerogenic index $(0.82$ and 0.89) compared with indomethacin and celecoxib. In molecular docking studies, interaction is excellent with receptors [25].

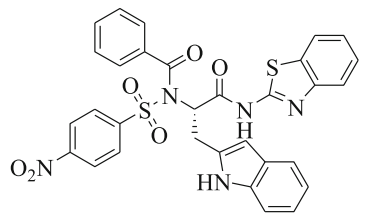

(42)

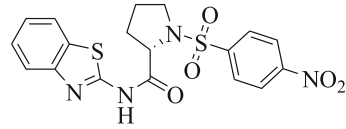

(43)
Indomethacin analogs of 2-(4-(methylsulfonyl)phenyl)-1-substituted-indole were synthesized by Shaker et al. and assessed for their in vitro COX-2 inhibitory act as well as in vivo anti-inflammatory activity. COX inhibitory activity (in vitro) evaluation showed selective binding with receptor $(\mathrm{COX}-2)$ with $\mathrm{SI}=$ 30.35-107.63 as compared to standard drug (SI = $0.079)$ whereas in vivo anti-inflammatory activity studies reported compounds 1-(4-chlorobenzyl)-2-(4(methylsulfonyl)phenyl)-1H-indole (44) (90.5\%), 1-(4chlorobenzyl)-5-methyl-2-(4-(methylsulfonyl)phenyl)$1 H$-indole (45) (75.6\%), 1-(4-chlorobenzyl)-5-fluoro2-(4-(methylsulfonyl)phenyl)- $1 H$-indole (46), and $(81.1 \%)$ as most active. Molecular modeling studies of the compounds 44 and 46 showed excellent binding interaction to COX-2 enzyme [26].

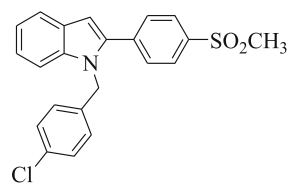

(44)

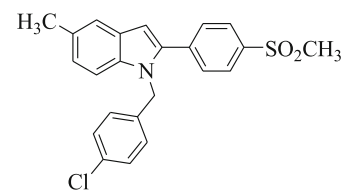

(44)

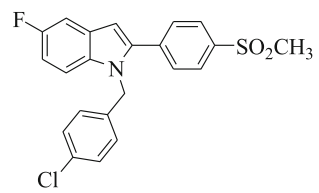

(46)

\section{Anticancer activity}

Zhuang et al. reported a series of 2, 4-disubstituted furo $[3,2-b]$ indoles for anticancer activity against the (human NCI-60) tumor cell lines. Among the tested compounds, compound (5-((2-)(hydroxymethyl)-4H-furo[3,2-b] indol-4-yl)methyl)furan-2-

yl)methanol (47) demonstrated the best anticancer activity. The analysis of results suggests that the fingerprint of the compound 48 is similar NSC754549 [27].

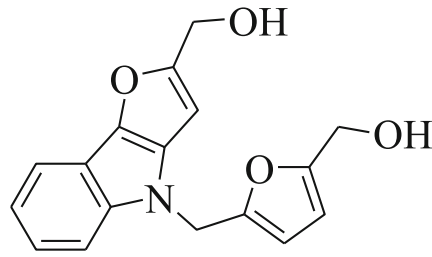

(47)

Indole-based derivatives of benzothiazole designed by Ma et al. showed antitumor activity against cancer cells breast MDA-MB-231, lung cells (H460), and colon cells (HT29) in MTT assay with positive controls as PAC-1 and oncrasin-1. Compound (E)-1-((1-(4-chlorobenzyl)$1 H$-indol-3-yl $)$ methylene)-4-(6-((dimethylamino) methyl) benzo $[d]$ thiazol-2-yl) semicarbazide (48) showed greatest antitumor activity [28].<smiles>CN(C)Cc1ccc2nc(NC(=O)NC=N)sc2c1</smiles>

(48)

Novel (E)-3-(5-substituted-1H-indol-3-yl)-1-(5, 5, 8, 8tetramethyl-5, 6, 7, 8-tetrahydronaphthalen-2-yl) prop-2en-1-one derivatives were prepared and examined for their anticancer effects by Gurkan-Alp et al. Compound (E)-3-(1H-indol-3-yl)-1-(5,5,8,8-tetramethyl-5,6,7,8-tetrahydronaphthalen-2-yl)prop-2-en-1-one (49) was found to be the most active [29].<smiles>CC1(C)CCC(C)(C)c2cc(C(=O)/C=C/c3c[nH]c4ccccc34)ccc21</smiles>

(49)

2,3-Dimethylindoles and tetrahydrocarbazoles also show anticancer properties against the cancer cell lines such as MCF10A, Calu1, HCT116, Panc1, ACHN, and $\mathrm{H} 460$ by using staining assay of propidium iodide (PI) as reported by Kumar et al. Compounds 2,3-dimethyl- $1 \mathrm{H}$-indole (50) and 5-fluoro-2,3dimethyl- $1 H$-indole (51) were found to be cytotoxic against cancer cell lines [30]. 
<smiles>Cc1[nH]c2ccccc2c1C</smiles>

(50)<smiles>Cc1[nH]c2ccc(F)cc2c1C</smiles>

(51)
5-(2-Carboxyethenyl) indole derivatives gave anticancer response against HT-29 and K562 cell lines as explained by Han et al. and compounds $(E)$-methyl 3-(1tolyl-1H-indol-5-yl)acrylate (52), (E)-methyl 3-(1-benzyl$1 H$-indol-5-yl)acrylate (53) and (E)-methyl 3-(1-(4-(trifluoromethyl)benzyl)- $1 H$-indol-5-yl)acrylate (54) demonstrated notable activity in cell lines(HT-29) with potency $4.67,8.24$ and $6.73 \mu \mathrm{M}$, respectively [31].

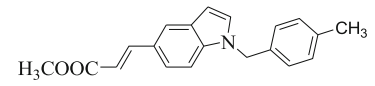

(52)

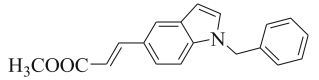

(53)<smiles>CC(=O)C=CCc1ccc2c(ccn2Cc2ccc(C(F)(F)F)cc2)c1</smiles>

3-[(4-Substitutedpiperazin-1-yl) methyl]-1H-indole derivatives were prepared using Mannich reaction by Akkoc et al. and evaluated for cytotoxic activity. The cytotoxicity of the compound was dependent on three cell lines: human liver (HUH7), breast (MCF7), and colon (HCT116). The most potent compound against cancer cell lines was 3-((4-(3,4dichlorobenzyl)piperazin-1-yl)methyl)- $1 H$-indole (55) [32].<smiles>Clc1ccc(CN2CCN(Cc3c[nH]c4ccccc34)CC2)cc1Cl</smiles>

(55)

3, 5-Bis (indolyl)-1, 2, 4-thiadiazoles showed cytotoxicity against selected human cancer cell lines reported by Kumar et al. and compound 1-(4-chlorobenzyl)-3-(5-(1-(4-chlorobenzyl)-4-methoxy-1 $H$-indol-3-yl)-1,2,4-thiadiazol-3-yl)-4methoxy- $1 H$-indole (56) gave the most potent activity (anticancer) [33].<smiles>COc1cccc2c1c(-c1nsc(-c3cn(Cc4ccc(Cl)cc4)c4cccc(OC)c34)n1)cn2Cc1ccc(Cl)cc1</smiles>

(56)
$\mathrm{N}-1$ and C-3 substituted indole derivatives also showed cytotoxic properties as reported by Choppara et al. Compounds (Z)-1-((5-bromo-1-(3-methylbut-2-enyl)- $1 H$ indol-3-yl)methylene)semicarbazide (57) and (Z)-1-((5bromo-1-(3-methylbut-2-enyl)-1 $H$-indol-3-yl)methylene)thiosemicarbazide (58) were found to be cytotoxic [34].

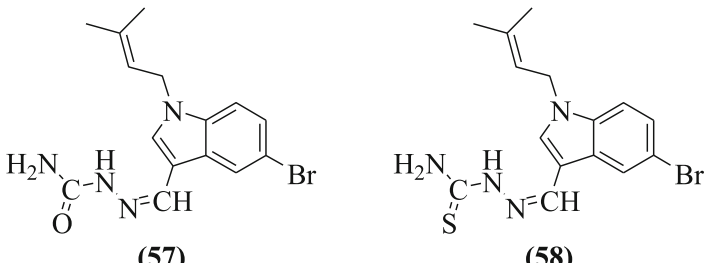

Indole hybridized diazenyl derivatives ${ }^{(\mathbf{5 8}}$ were designed and reported for cytotoxicity against human cell lines, i.e., leukemic cell (K562), normal cell (HEK293), lung cell (HCT-116), and breast cell (MDAMB231) adopting MTT assay by Kaur et al. Compounds $(R)-5-(((E)-1-(4-$ ((Z)-(1H-indol-3-yl)diazenyl)phenyl)ethylidene)amino)cyclohexa-2,4-diene-1-carboxylic acid (59) and $\mathrm{N}$-(2(((E)-1-(4-((Z)-(1H-indol-3-yl)diazenyl)phenyl)ethylidene)amino)ethyl)naphthalen-2-amine (60) showed potential against breast cancer cell line (MDAMB231) [35].

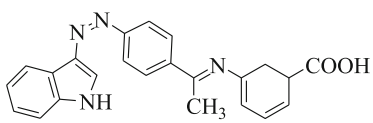

(59)

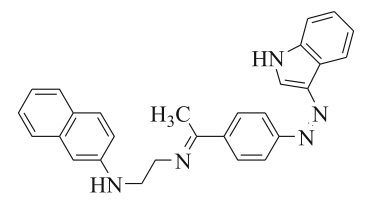

(60)
The derivatives of 2-phenylindole containing triazine, thiazolo-s-triazine, imidazole sugar, imidazolothiazole, and imidazole were prepared and screened for their anticancer activity against colorectal carcinoma, liver carcinoma, prostate cancer, and breast adenocarcinoma by Yousif et al. The compounds 2-chloro-1-(2-phenyl-1H-indol-1-yl)ethanone (61), 4-(2-phenyl-1H-indol-1-yl)- $1 H$-imidazol-2(5H)-one (62) and ethyl 2-(2-oxo-4-(2-phenyl- $1 H$-indol-1-yl)-2H-imidazol$1(5 H)$-yl)acetate (63) showed high cytotoxic activity [36].

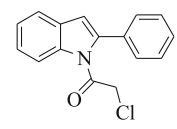

(61)

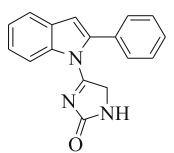

(62)

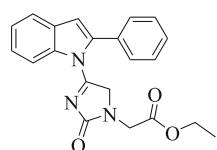

(63)

\section{Anti-HIV activity}

Kasralikar et al. reported a series of novel indolyl and oxochromenyl xanthenone derivatives and performed their molecular docking studies as an anti-HIV-1. In tested compounds, compounds 9-(1H-indol-3-yl)-7methoxy-3,3-dimethyl-3,4-dihydro- $2 H$-xanthen-1(9H)- 
one (64), 9-(1H-indol-3-yl)-3,3-dimethyl-3,4-dihydro$2 H$-xanthen-1 $(9 H)$-one $(65)$ and 7-bromo-9-(1H-indol-3yl)-3,3-dimethyl-3,4-dihydro- $2 H$-xanthen-1(9H)-one (66) were found to be most active compounds [37].

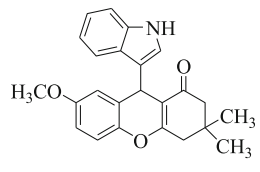

(64)

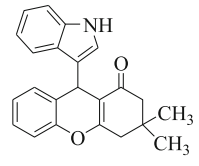

(65)<smiles>CC1(C)CC(=O)C2=C(C1)Oc1ccc(Br)cc1C2c1cccc2[nH]ccc12</smiles>

$N$-arylsulfonyl-3-acetylindole derivative was prepared and evaluated as HIV-1 inhibitors analogs by Ran et al. Compounds 1-(4-methyl-1-(phenylsulfonyl)- $1 H$-indol-3yl)ethanone (67) and 1-(1-(4-ethylphenylsulfonyl)-4-methyl- $1 H$-indol-3-yl)ethanone (68) were the most effective against the anti-HIV-1 activity. SAR showed that acetyl group derivatives were more active [38].
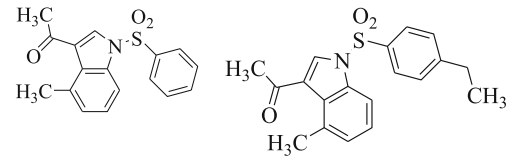

4-[(1,2-Dihydro-2-oxo-3H-indol-3-ylidene)amino] $N(4,6$-dimethyl-2-pyrimidinyl)-benzene derivatives were synthesized and screened for their anti-HIV activity against HIV-1 (IIIB) and HIV-2 (ROD) strains replication in acutely infected cells (MT-4) by Selvam et al. Compounds (Z)-3-(4-((4,6-dimethylpyrimidin-2-ylamino)methylsulfonyl)phenylimino)indolin-2one (69), (Z)-5-chloro-3-(4-((4,6-dimethylpyrimidin-2-ylamino)methylsulfonyl)phenylimino)indolin-2-one (70), (Z)-5bromo-3-(4-((4,6-dimethylpyrimidin-2-ylamino)methylsulfonyl)phenylimino)indolin-2-one (71), (Z)-3-(4-((4,6-dimethylpyrimidin-2-ylamino)methylsulfonyl)phenylimino)-5-methylindolin-2-one (72) and (Z)-1-acetyl-3-(4-((4,6dimethylpyrimidin-2-ylamino)methylsulfonyl)phenylimino)Indolin-2-one (73) were found to be most effective [39].

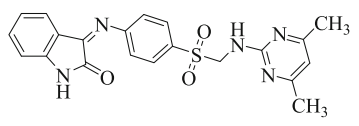

(69)

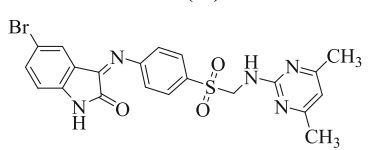

(71)

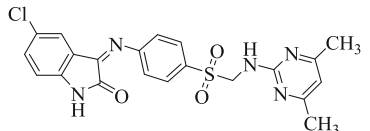

(70)

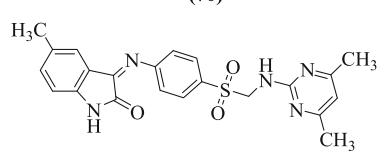

(72)<smiles>CC(=O)N1C(=O)/C(=N\c2ccc(S(=O)(=O)CNc3nc(C)cc(C)n3)cc2)c2ccccc21</smiles>

(73)
$\mathrm{N}$-Arylsulfonyl-3-acylindole benzoyl hydrazone derivatives were reported as HIV-1 inhibitors by Che et al. Among the reported analogs, compounds $(E)-N^{\prime}-(1-(1-$ (3-nitrophenylsulfonyl)-1 $H$-indol-3-yl)ethylidene)benzohydrazide (74) and (E)-2-methyl- $N^{\prime}$-(1-(1-(3-nitrophenylsulfonyl)- $1 H$-indol-3-yl)ethylidene)benzohydrazide (75) displayed the highest $\mathrm{IC}_{50}$ and therapeutic index (TI) values $0.26,769.23$, and $0.31,645.16$ in microgram for anti-HIV-1 respectively [40].
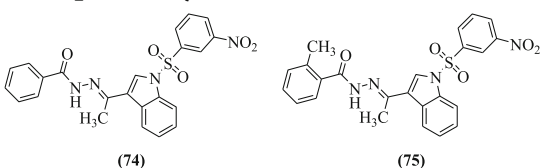

1-(Thiophen-2-yl)-9H-pyrido[3,4-b]indole derivatives were synthesized and screened for their anti-HIV activity by Ashok et al. and structure-activity relationship (SAR) studies stated that electron-withdrawing group and electron-donating ortho, para directing groups increases the antiviral activities. Among the synthesized derivatives, derivative (4-(3-methoxyphenyl)piperazin-1-yl)(1(thiophen-2-yl)-9H-pyrido[3,4-b]indol-3-yl)methanone (76) showed significant anti-HIV activity with selectivity index (SI) 483 and $\mathrm{IC}_{50}(0.53 \mu \mathrm{M})$. Lipinski rule is followed by these compounds in the molecular predication studies (In-silico) [41].<smiles>COc1cccc(N2CCN(C(=O)c3cc4c([nH]c5ccccc54)c(-c4cccs4)n3)CC2)c1</smiles>

(76)

Indole-based reverse transcriptase inhibitors (non-nucleoside) were prepared and tested for anti-HIV virus type HIV-1 $1_{\text {IIIB }}$ using TZM-bl cell assay by Han et al. SAR studies showed that substituent affects the potency. From the synthesized compounds, compounds methyl 2amino-3, 3, 3-trifluoro-2-(6-fluoro- $1 H$-indol-3-yl) propanoate (77) and methyl 3-(2-amino-3-ethoxy-1, 1, 1trifluoro-3-oxopropan-2-yl)-1 $H$-indole-5-carboxylate (78) were most active with $\mathrm{IC}_{50}$ values $(0.060 \mu \mathrm{M}$ and $0.045 \mu \mathrm{M}$ respectively) [42].<smiles>CCOC(=O)C(N)(c1c[nH]c2ccc(C(C)=O)cc12)C(F)(F)F</smiles>

(77)<smiles>COC(=O)C(N)(c1c[nH]c2cc(F)ccc12)C(F)(F)F</smiles>

(78)
2-(1H-Indol-3-yl) ethylthiourea derivatives were explained as anti-HIV agents by Sanna et al. The 
compound 1-(2-(1H-indol-3-yl)ethyl)-3-(4-bromophenyl)thiourea (79) showed potent activity against HIV-1 [43].

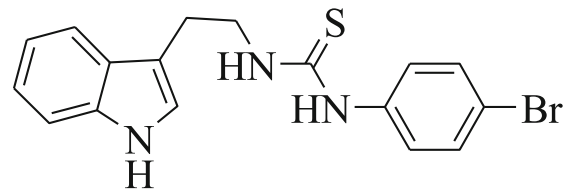

(79)

Indolyl aryl sulfones were discussed through molecular modeling studies using 3-D QSAR model as new antiHIV agents by Ragno et al. From the screened compounds, compounds 5-chloro-3-(o-tolylsulfonyl)- $1 \mathrm{H}$-indole-2-carbohydrazide (80) and 5-chloro- $\mathrm{N}^{\prime}$-isopropyl-3(o-tolylsulfonyl)- $1 H$-indole-2-carbohydrazide (81) were found to be the most potent compound against C-8166 and MT-4 cell [44].<smiles>Cc1ccccc1S(=O)(=O)c1c(C(=O)NN)[nH]c2ccc(Cl)cc12</smiles>

(80)<smiles>Cc1ccccc1S(=O)(=O)c1c(C(=O)NNC(C)C)[nH]c2ccc(Cl)cc12</smiles>

(81)
Ethyl 6-bromo-5-hydroxy- $1 H$-indole-3-carboxylates derivatives were prepared and investigated for anti-hepatic activities by Chai et al. Compounds ethyl 6-bromo-1-cyclopropyl-2((3,4-difluorophenylsulfinyl)methyl)-5-hydroxy-4-(1H-imidazol-1-yl)- $1 H$-indole-3-carboxylate (82), ethyl 6-bromo-2-((3, 4-difluorophenylsulfinyl)methyl)-5-hydroxy-1-methyl-4-(5methyl-1H-imidazol-1-yl)- $1 H$-indole-3-carboxylate (83), ethyl 6-bromo-1-cyclopropyl-2-(((4-fluorophenyl)sulfinyl)methyl)4-(((guanidinoselanyl)(imino)methyl)amino)-5-hydroxy- $1 H$ indole-3-carboxylate (84), and ethyl 6-bromo-1-cyclopropyl2-((3,4-difluorophenylsulfinyl)methyl)-5-hydroxy-4-(4methylpiperazin-1-yl)- $1 H$-indole-3-carboxylate (85) possessed significant activities with $\mathrm{IC}_{50}(3.6,6.37,5.2$, and $5.4 \mu \mathrm{g} / \mathrm{ml}$ ) against hepatitis B virus (HBV) [45].<smiles>CCOC(=O)c1c(C(=O)CSc2ccc(F)c(F)c2)n(C2CC2)c2cc(Br)c(O)c(-n3ccnc3)c12</smiles>

(82)

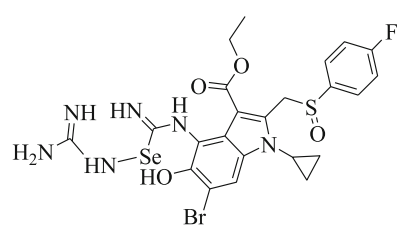

(83)

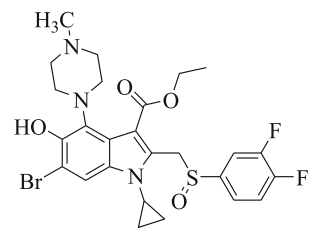

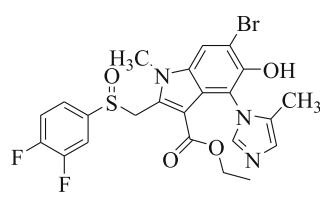

Ethyl 5-hydroxy-1H-indole-3-carboxylates derivatives were also explained for their hepatitis inhibitory activities in cells by Zhao et al. The structures ethyl 1cyclopropyl-5-hydroxy-4-((5-methyl- $1 H$-imidazol-1yl)methyl)-2-(phenylsulfonylmethyl)- $1 H$-indole-3-carboxylate (86) and ethyl 5-hydroxy-2-((3-methoxyphenylsulfonyl)methyl)-1-methyl-4-(pyrrolidin-1-ylmethyl)- $1 H$ indole-3-carboxylate (87) exhibited significant activity against hepatitis $\mathrm{B}$ virus (HBV) with the $\mathrm{IC}_{50}$ values of compounds $(24.90 \mu \mathrm{g} / \mathrm{ml})$ and $(15.41 \mu \mathrm{g} / \mathrm{ml})$ higher than those of the used of reference drug lamivudine $(228.00 \mu \mathrm{g} / \mathrm{ml})$ [46].

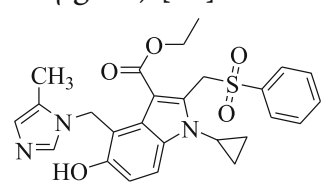

Heterocycle-containing oxindoles derivatives were pre-

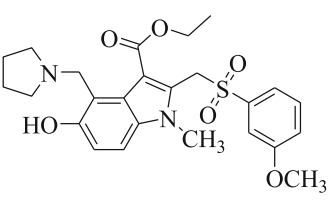

Heterocycle-containing oxindoles derivatives were prepared and evaluated for their anti-HIV activity by Jiang et al. In this series, compound $(1 S, 2 S)$-5'-chloro-2-(pyridin-2-yl)spiro[cyclopropane-1,3'-indolin]-2'-one (88) exhibited potent inhibitory activities against viruses [47].<smiles>O=C1Nc2ccc(Cl)cc2C12CC2c1ccccn1</smiles>

(88)

Indole sulfonamides derivatives were designed and evaluated as non-nucleoside reverse transcriptase inhibitors (NNRTIs) by Zhao et al. Among all, the analogs $N$ (2-chloro-6-fluorobenzyl)-5-bromo-3-(pyrrolidin-1-ylsulfonyl)- $1 H$-indole-2-carboxamide (89), $N$-(2-hydroxybenzyl)-5-bromo-3-(pyrrolidin-1-ylsulfonyl)- $1 H$-indole-2-

carboxamide (90), and 5-bromo-2-(5-methyl- $1 \mathrm{H}$-imidazol-2-yl)-3-(pyrrolidin-1-ylsulfonyl)- $1 H$-indole (91) improved the activities against HIVRT mutants Y181C and K103N which retain potent cellular activity [48].

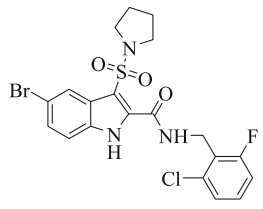

(89)

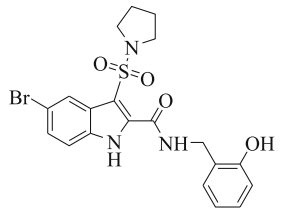

(90)

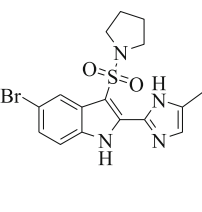

(91)
(R)-N-(3-(1H-indol-5-yloxy)-2-hydroxypropyl)-N-isobutyl benzenesulfonamide derivatives were screened for anti-HIV activity by Chiummiento et al. Among all compounds in this series, compounds $(R)-N-(3-(1 H$-indol-5yloxy)-2-hydroxypropyl)- $N$-isobutyl-4nitrobenzenesulfonamide (92), ( $R$ )- $N$-(3-(1H-indol-5-yloxy)-2-hydroxypropyl)- $N$-isobutyl-3,4-dimethoxybenzenesulfonamide (93), 
and $(R)-N$-(3-(1H-indol-5-yloxy)-2-hydroxypropyl)-4amino- $N$-isobutylbenzenesulfonamide (94) demonstrated the best results [49].
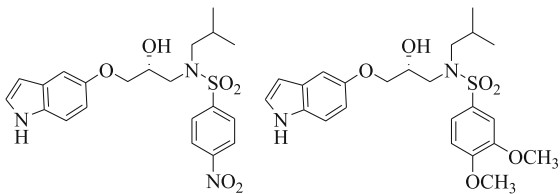

(92)

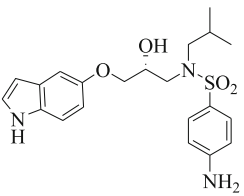

(94)
(E)-4-(1-(Substitutedbenzyl)-4-methoxy-1H-indol-3yl)-2-hydroxy-4-oxobut-2-enoic acid derivatives were reported as new HIV inhibitor (integrase strand-transfer inhibitors) by Ferro et al. The binding modes of the compounds were studied by induced-fit docking (IFD). Among all compounds, (E)-4-(1-(4-chlorobenzyl)-4methoxy-1 $H$-indol-3-yl)-2-hydroxy-4-oxobut-2-enoic acid (95), (E)-4-(1-(3-chloro-5-fluorobenzyl)-4-methoxy$1 H$-indol-3-yl)-2-hydroxy-4-oxobut-2-enoic acid (96), and (E)-4-(1-(4-chloro-3-fluorobenzyl)-4-methoxy-1 $H$ indol-3-yl)-2-hydroxy-4-oxobut-2-enoic acid showed inhibition (in strand-transfer) in respect to elvitegravir [50].<smiles>COc1cccc2c1c(C(=O)C=C(O)C(=O)O)cn2Cc1ccc(Cl)cc1</smiles>

(95)

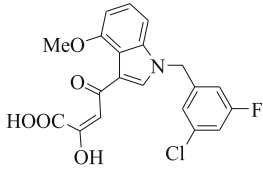

(96)

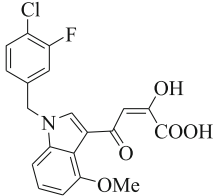

(97)

\section{Antioxidant activity}

$\mathrm{Di}(1 H$-indol-3-yl $)$ sulfane derivatives were prepared and evaluated as antioxidants agents by Silveira et al. Compound di( $1 H$-indol-3-yl)sulfane (98) exhibited antioxidant activity in ferric reducing ability of plasma (FRAP), 2,2-Diphenyl-1-picrylhydrazyl (DPPH) and 2,2'-Azinobis(3-ethylbenzothiazoline-6-sulfonic acid ) (ABTS) assays at micromolar concentration [51].<smiles>c1ccc2c(Sc3c[nH]c4ccccc34)c[nH]c2c1</smiles>

(98)

(E)-1-(Substitutedphenyl)-2-((2-(4-fluorophenyl)-1Hindol-3-yl)methylene)hydrazine derivatives as MLT (Melatonin) analogs were prepared by Gurer-Orhan et al. and were tested for antioxidant activity in human erythrocytes. Compounds (E)-1-(2-chlorophenyl)-2-((2(4-fluorophenyl)- $1 H$-indol-3-yl)methylene)hydrazine

(99), (E)-1-(4-chlorophenyl)-2-((2-(4-fluorophenyl)-1Hindol-3-yl)methylene)hydrazine (100), (E)-1-(3-bromophenyl)-2-((2-(4-fluorophenyl)-1H-indol-3- yl)methylene)hydrazine (101), and (E)-1-(2-fluorophenyl)-2-((2-(4-fluorophenyl)-1H-indol-3-yl)methylene)hydrazine (102) exhibited significant activity [52].

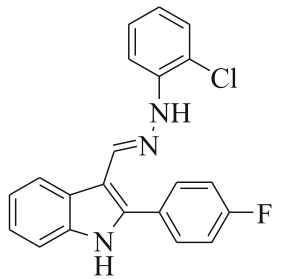

(99)<smiles>Fc1ccc(-c2[nH]c3ccccc3c2/C=N/c2cccc(Br)c2)cc1</smiles>

(101)<smiles></smiles>

(100)<smiles>N=Cc1c(-c2ccc(F)cc2)[nH]c2ccccc12</smiles>

(102)
(E)-5-(1-(Substitutedphenyl)-3-(1H-indol-3-yl) allylidene) pyrimidine-2, 4, $6(1 H, 3 H, 5 H)$-trione derivatives containing barbitone moiety were reported as DNA cleavage and antioxidant agents by Biradar et al. The compounds $(E)-5$-(3-(1H-indol-3-yl)-1-phenylallylidene)pyrimidine-2,4,6 $(1 H, 3 H, 5 H)$-trione (103), $(E)-5-(3-(1 H-$ indol-3-yl)-1-p-tolylallylidene)pyrimidine-2,4,6(1H,3H, $5 H$ )-trione (104) and (E)-5-(1-(4-chlorophenyl)-3-(1Hindol-3-yl)allylidene)pyrimidine-2,4,6(1H,3H,5H)-trione (105) exhibited excellent antioxidant and DNA cleavage activities [53].

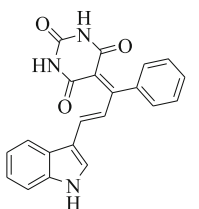

(103)

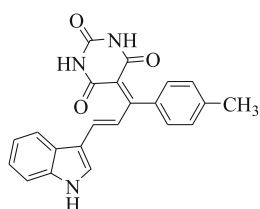

(104)

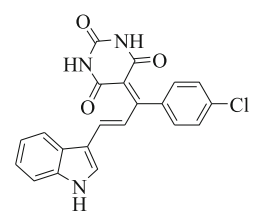

(105)
Indole derivatives like tryptophan and tryptamine were investigated for DNA clevaging activity by Estevão et al. Compounds 2-amino-3-(1-(3-methylbut-2-enyl)- $1 \mathrm{H}$ indol-3-yl)propanoic acid (106) $\left(\mathrm{IC}_{50} 4.13 \pm 0.17 \mu \mathrm{M}\right), 2$ amino-3-(2-(3-methylbut-2-enyl)-1 $H$-indol-3-yl)propanoic acid (107) $\left(\mathrm{IC}_{50} 4.56 \pm 0.48 \mu \mathrm{M}\right)$, and methyl 2-(1,3dioxoisoindolin-2-yl)-3-(2-isopentyl- $1 H$-indol-3-yl)propanoate $(108)\left(\mathrm{IC}_{50} 14.0 \pm 6.8 \mu \mathrm{M}\right)$ showed significant activity [54].

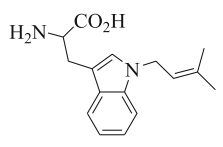

(106)

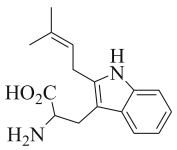

(107)

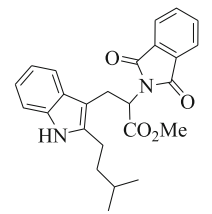

(108) 
(E)-1-((2-Phenyl-3H-inden-1-yl) methylene)-4-substitutedthiosemicarbazides, a new class of antioxidant agents, were synthesized by Bakherad et al. and compounds exhibited better anti-oxidant activities. Compound (E)-1-((2-phenyl-3H-inden-1-yl)methylene)-4- $p$ tolylthiosemicarbazide (109) found to be the most potent compound [55].

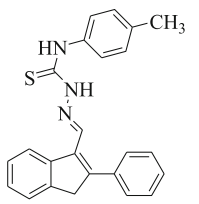

(109)

(E)-1-(Substitutedphenyl)-2-((1-methyl-1H-indol-2yl)methylene)hydrazines were synthesized and reported as antioxidant agents by Suzen et al., and compounds (E)-1-(2,5-difluorophenyl)-2-((1-methyl-1 $H$-indol-2yl)methylene)hydrazine (110), (E)-1-(2,5-dichlorophenyl)-2-((1-methyl-1H-indol-2-yl)methylene)hydrazine (111) and (E)-1-(2,6-dichlorophenyl)-2-((1-methyl-1Hindol-2-yl)methylene)hydrazine (112) were the most promising compounds [56].

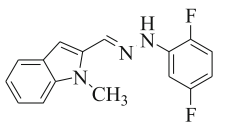

(110)

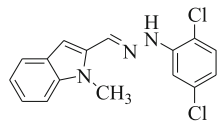

(111)

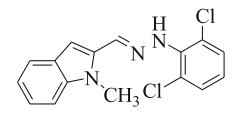

(112)
5-Chloroindole hydrazide/hydrazone derivatives were prepared and evaluated for antioxidant activity by Yllmaz et al. 2,2-Diphenyl-1-picrylhydrazyl (DPPH) radical scavenging activity ( $\mathrm{IC}_{50} 2$ to $\left.60 \mu \mathrm{M}\right)$ was shown by all compounds. Compound (E)-1-((5-chloro- $1 H$-indol-2-yl) methylene)-2-(2-chlorophenyl) hydrazine (113) possessed high inhibitory activities in assay (LP) against melatonin at $0.1 \mathrm{mM}[57]$.<smiles>Clc1ccc2[nH]c(/C=N/Nc3ccccc3Cl)cc2c1</smiles>

(113)

3-(1-(4-(4-chlorophenyl)thiazol-2-yl)-3-(substitutedphenyl)- $1 H$-pyrazol-5-yl)- $1 H$-indole derivatives were reported as antioxidant agents by Ummadi et al. Compounds 3-(1-(4-(4-chlorophenyl)thiazol-2-yl)-3-ptolyl-1H-pyrazol-5-yl)- $1 H$-indole (114) and 3-(1-(4-(4chlorophenyl)thiazol-2-yl)-3-(4-methoxyphenyl)-1H-pyrazol-5-yl)-1H-indole (115) showed excellent antioxidant activity in comparison to ascorbic acid. Structural activity relationship (SAR) stated that $\mathrm{OCH}_{3}, \mathrm{CH}_{3}$ groups showed higher activity (scavenging) as compared to $\mathrm{NO}_{2}, \mathrm{Cl}, \mathrm{Br}$ groups [58].

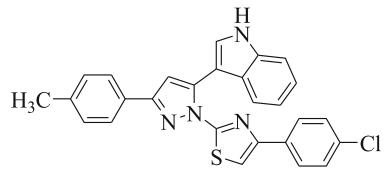

(114)

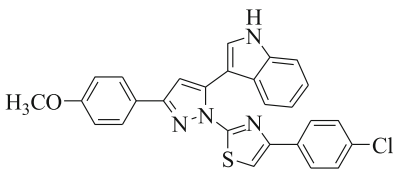

(115)
Melatonin retinamide derivatives were synthesized through reaction between the melatonin and tetrahydrotetramethylnaphthalene carboxylic acid and screened for antioxidant activity by Ates-Alagoz et al. The compounds have weak 2,2-Diphenyl-1-picrylhydrazyl (DPPH) inhibition activity pattern and some of the compounds $N$-(2-(1H-indol-3-yl)ethyl)-5,5,8,8-tetramethyl-5, 6,7,8-tetrahydronaphthalene-2-carboxamide (116), $\mathrm{N}$-(2(5-methoxy-1H-indol-3-yl)ethyl)-5,5,8,8-tetramethyl-5,6, 7,8-tetrahydronaphthalene-2-carboxamide (117), $\mathrm{N}-(1 \mathrm{H}$ indol-5-yl)-5,5,8,8-tetramethyl-5,6,7,8-tetrahydronaphthalene-2-carboxamide (118), $\quad N$-(3,5,5,8,8-pentamethyl-5,6,7,8-tetrahydronaphthalen-2-yl)- $1 H$-indole-2carboxamide (119), 4-(1H-indol-3-yl)- $N$-(3,5,5,8,8-pentamethyl-5,6,7,8-tetrahydronaphthalen-2-yl)butanamide (120), and 5-(1H-indol-3-yl)- $N$-(3,5,5,8,8-pentamethyl-5, 6,7,8-tetrahydronaphthalen-2-yl)pentanamide (121) possessed a strong inhibition of lipid peroxidation $(88,96$, 90, 94, 93, and 86\%) [59].
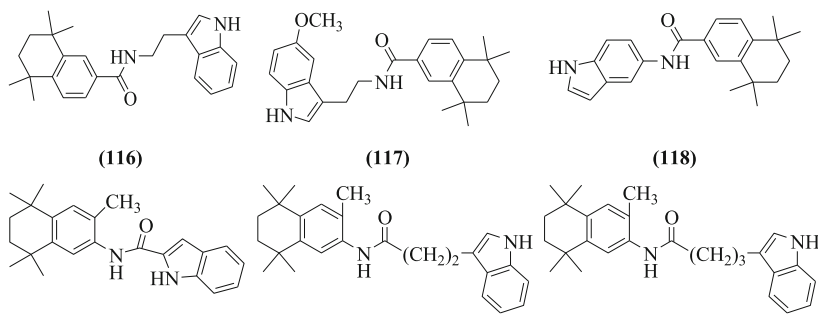

(119)

(120)

(121)

$N$-((10-nitro-1H-indolo $\quad[1, \quad 2-c]$ quinazolin-12$y l$ )methylene)benzenamines were prepared and tested for their anti-oxidant activity by Dixit et al. Nearly all derivatives have shown good antioxidant activity at all the concentrations, but compounds 4-nitro- $N-((10-$ nitro $H$-indolo[1,2-c]quinazolin-12-yl)methylene)benzenamine (122), 4-fluoro- $N$-((10-nitro $H$-indolo[1,2-c] quinazolin-12-yl)methylene)benzenamine (123), and 4chloro-2-nitro- $N$-((10-nitro $H$-indolo[1,2-c] quinazolin12-yl)methylene)benzenamine (124) were found to be the best free radical scavengers [60].

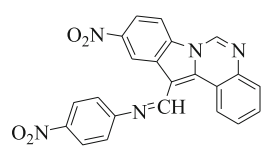

(122)

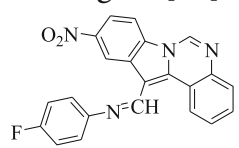

(123)

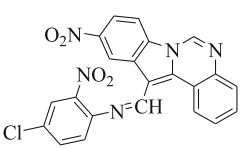

(124)

\section{Antimicrobial activity}

Indole[1,2-c]-1,2,4- butylnitrite benzotriazine derivatives were prepared using Sandmeyer reaction and screened 
for the antifungal activity by $\mathrm{Xu}$ et al. Compound indole[1,2-c]-1,2,4- butylnitrite benzotriazine (125) was more potent derivative [61].<smiles>c1ccc2c(c1)cc1nnc3ccccc3n12</smiles>

(125)

3-(2-(5-(Substitutedphenyl)-1, 3, 4-oxadiazol-2ylthio) ethylthio)-5-( $1 H$-indol-3-yl)-4H-1, 2, 4triazol-4-amines were prepared by Shi et al. using special technique, i.e., ultrasonic. Compounds 3-(2(5-(2-ethoxyphenyl)-1,3,4-oxadiazol-2-

ylthio)ethylthio)-5-(1H-indol-3-yl)-4H-1,2,4-triazol4-amine (126), and 3-(2-(5-(4-chlorophenyl)-1,3,4oxadiazol-2-ylthio)ethylthio)-5-( $1 H$-indol-3-yl)-4H-1, 2,4-triazol-4-amine (127) exhibited excellent activity against Staphylococcus aureus and Escherichia coli strains [62].

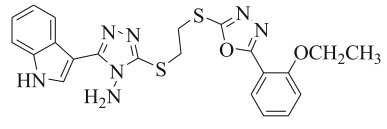

(126)

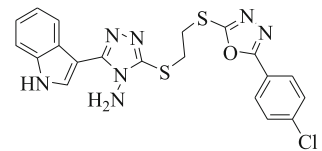

(127)
Substituted 1, 2, 3, 4-tetrahydropyrazino [1, 2-a] indole derivatives were reported by Tiwari et al. as antimicrobial agents against bacteria (both Gram positive and negative). Compounds 1-(4-fluorophenyl)-10-methyl-1,2,3,4-tetrahydropyrazino[1,2-a]indole (128), 10 methyl-1- $p$-tolyl-1,2,3,4-tetrahydropyrazino[1,2- $a]$ indole (129), and 10-methyl-1-(4-nitrophenyl)-1,2,3,4tetrahydropyrazino[1,2-a]indole (130) exhibited significant activity [63].

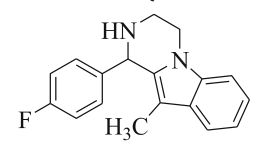

(128)

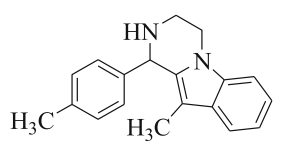

(129)<smiles>Cc1c2n(c3ccccc13)CCNC2c1ccc([N+](=O)[O-])cc1</smiles>

(130)

Methyl (E)-1-(substitutedbenzyl)-3-((2-(4, 5-dihydro-1Himidazol-2-yl)hydrazono)methyl)- $1 H$-indole-5carboxylate derivatives were reported as antimicrobial agents by Hong et al. Compounds (131), (132), (133), and (134) showed activity against Mycobacterium tuberculosis, multidrug-resistant Acinetobacter baumanii, and Gram-negative bacteria [64].

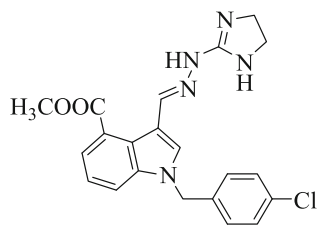

(131)

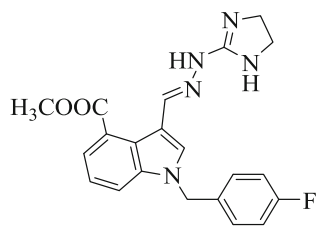

(133)

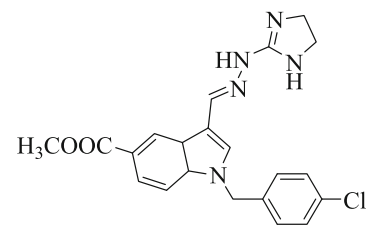

(132)

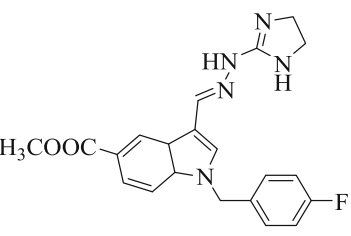

(134)
Azo dye of indoles were prepared by Ozturk et al and evaluated in vitro against yeast Saccharomyces cerevisiae, Gram (+), and (-) bacteria. Compounds (E)-ethyl 4- $((1 H-$ indol-3-yl) diazenyl) benzoate (135), (E)-ethyl 4-((1-methyl-1H-indol-3-yl) diazenyl) benzoate (136) and $(Z)-1$ (4-methoxyphenyl)-2-(3-methyl-1H-indol-2-yl)diazene (137) showed good activity [65].

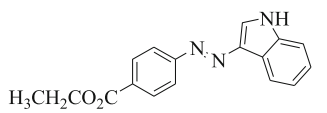

(135)

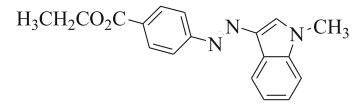

(136)

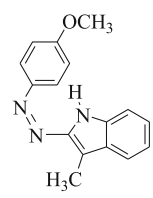

(137)

2, 3-Diarylindoles derivatives containing amine substituent at the 5 and 6 positions of indole were prepared and screened as anticoccidial agents by Scribner et al. Compound 2-(4-fluorophenyl)-6-(piperidin-4-yl)-3-(pyridin-3-yl)- $1 H$-indole (138) showed the best activity [66].<smiles>Fc1ccc(-c2[nH]c3cc(C4CCNCC4)ccc3c2-c2cccnc2)cc1</smiles>

(138)

A series of nitrogen and carbon substituted bisindoles were synthesized and investigated for antimicrobial agents by Singh et al. The $N$-benzyl moiety or 
morpholine or pyrrolidine at position 3 and xylidine or butane or propane as the bridge between the indoles were good for activity. Dockings studies showed strong interactions in the active sites of topoisomerase II lanosterol demethylase and dihydrofolate reductase. Compounds (139) and (140) were more active [67].

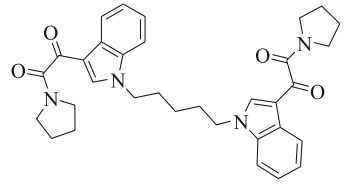

(139)

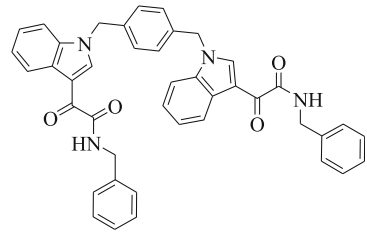

(140)

\section{Antitubercular activity}

(E)-1-(2-(1H-indol-3-yl)-5-(pyridin-4-yl)-1,3,4-oxadiazol$3(2 H)$-yl)-3-(substitutedphenyl)prop-2-en-1-one derivatives derived from pyridine and Indole were prepared and investigated in active and dormant state against $\mathrm{H}_{37} \mathrm{Ra}$ MTB (Mycobacterium tuberculosis) and BCG (Mycobacterium bovis) for their in vitro antitubercular activity by Desai et al. Compounds $(E)-1-(2-(1 H$-indol-3yl)-5-(pyridin-4-yl)-1,3,4-oxadiazol-3(2H)-yl)-3-(2-hydroxyphenyl)prop-2-en-1-one (141), (E)-1-(2-(1H-indol-3yl)-5-(pyridin-4-yl)-1,3,4-oxadiazol-3(2H)-yl)-3-(2-nitrophenyl)prop-2-en-1-one (142), (E)-1-(2-(1H-indol-3-yl)5-(pyridin-4-yl)-1,3,4-oxadiazol-3(2H)-yl)-3-(4-nitrophenyl)prop-2-en-1-one (143), and (E)-1-(2-(1H-indol-3-yl)5-(pyridin-4-yl)-1,3,4-oxadiazol-3(2H)-yl)-3-(2,4-dichlorophenyl)prop-2-en-1-one (144) exhibited effective antitubercular activity [68].

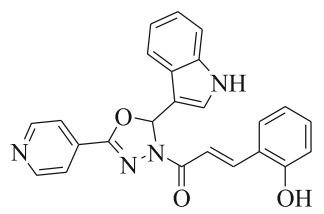

(141)<smiles>O=C(/C=C/c1ccc([N+](=O)[O-])cc1)c1nc(-c2ccncc2)oc1-c1ccccc1</smiles>

6-Cyano-5-methoxyindolo $[2,3-a]$ carbazole derivatives were designed and screened for their moderate inhibitory activities against $\mathrm{H} 37 \mathrm{Rv}$ (Mycobacterium tuberculosis) strain and Bacillus anthracis (ANR) strain by Guo et al. to combat tuberculosis and anthrax infections. Compounds 6-methoxy-11,12-dihydroindolo[2,3a] carbazole-5-carbonitrile (145), 11,12-dihydroindolo[2, 3-a]carbazole-5-carbonitrile (146), and 11-benzyl-11,12dihydroindolo[2,3-a] carbazole-5-carbonitrile displayed moderate activity against Mycobacterium tuberculosis and good activity against Bacillus anthracis [69].<smiles></smiles>

(145)<smiles></smiles>

(146)<smiles>N#Cc1cc2c3ccccc3[nH]c2c2ccccc12</smiles>

(147)
Indole and pyridine nuclei combined through hydrazones-hydrazide and hydrazides were evaluated their in vitro antimycobacterial activity by Velezheva et al. In these series, the most potent compound was ethyl (E)-3-((2-isonicotinoylhydrazono)methyl)-5-methyl- $1 H$-indole-2-carboxylate (148) (MIC value of $0.05 \mu \mathrm{g} / \mathrm{mL}$ and selectivity index 300) was active against Mycobacterium tuberculosis $\mathrm{H}_{37} \mathrm{Rv}$ [70].<smiles>CCOC(=O)c1[nH]c2ccc(C)cc2c1/C=N\NC(=O)c1ccncc1</smiles>

(148)

Walmik et al. synthesized several novel $N^{\prime}-((2-$ phenyl-1H-indol-3-yl) methylene), substituted phenyl$1 \mathrm{H}$-indole-2-carbohydrazide derivatives, and screened for their in vitro antimycobacterial activity. The antitubercular result showed that chlorine derivatives were most active. Compound 5-chloro-3-phenyl- $N^{\prime}$ ((2-phenyl-1 $H$-indol-3-yl)methylene)- $1 H$-indole-2-carbohydrazide (149) $(\mathrm{MIC}=0.2 \mu \mathrm{g} / \mathrm{mL})$ possessed potent growth inhibitory effect against $\mathrm{H}_{37} \mathrm{Rv}$ Mycobacterium tuberculosis [71].<smiles>N=Cc1c(-c2ccccc2)[nH]c2ccccc12</smiles>

(149)

3-(1-Isonicotinoyl-3-(subtituted-2-phenyl- $1 H$-indol-3yl)-1H-pyrazol-5-yl)-2H-chromen-2-one derivatives were synthesized and reported as antimycobacterial agents by Rathod et al. In molecular docking studies, the mode of action of these active derivatives were studied and compounds 3-(1-isonicotinoyl-3-(5-methyl-2-phenyl- $1 \mathrm{H}$ - 
indol-3-yl)- $1 H$-pyrazol-5-yl)-2 $H$-chromen-2-one (150) and 3-(3-(1H-indol-3-yl)-1-isonicotinoyl- $1 H$-pyrazol-5yl)-2H-chromen-2-one (151) gave the promising effect with Mycobacterium tuberculosis $\mathrm{H}_{37} \mathrm{Rv}$ strain at 12.5 and $25 \mu \mathrm{g} / \mathrm{ml}$ respectively [72].<smiles>Cc1ccc2[nH]c(-c3ccccc3)c(-c3cc(-c4cc5ccccc5oc4=O)n(C(=O)c4ccncc4)n3)c2c1</smiles>

(150)

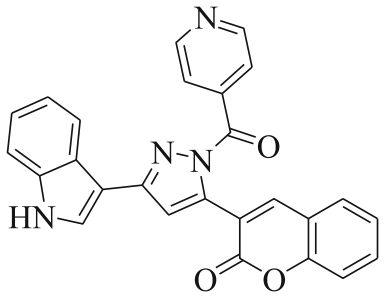

(151)
$N$-(4, 4-dimethylcyclohexyl)-substitutedindole-2-carboxamides were reported as antituberculosis agents by Kondreddi et al. Structure-activity relationship ( SAR) studies revealed that alkyl groups reduced solubility and increased Mycobacterium tuberculosis activity. Compounds 4,6-dichloro- $\mathrm{N}$-(4,4-dimethylcyclohexyl)- $1 \mathrm{H}$-indole-2-carboxamide (152) and N-(4,4dimethylcyclohexyl)-4,6-difluoro- $1 H$-indole-2-carboxamide (153) active compounds displayed improved in vitro activity [73].<smiles>CC1(C)CCC(NC(=O)c2cc3c(Cl)cc(Cl)cc3[nH]2)CC1</smiles>

(152)<smiles>CC1(C)CCC(NC(=O)c2cc3c(F)cc(F)cc3[nH]2)CC1</smiles>

(153)
Spirothiazolidinone derivatives of 5-chloro-3-phenyl$1 H$-indoles were synthesized by Cihan-Üstündağ et al. and evaluated for their in vitro antitubercular activity. Among all, compounds 5-chloro-3-phenyl-N-(8-phenyl3-oxo-1-thia-azaspiro[4.5]-decan-4-yl)-3-phenyl-1H-indole-2-carboxamide (154) $(\mathrm{MIC}=3.9 \mu \mathrm{M})$ and 5-chloro$\mathrm{N}$-(2-methyl-8-phenyl-3-oxo-1-thia-4-azaspiro[4.5]-decan-4-yl)-3-phenyl-1H-indole-2-carboxamide $(\mathrm{MIC}=7.8 \mu \mathrm{M})$ were the most active compounds having 8-phenyl spiro ring against $\mathrm{H}_{37} \mathrm{Rv}$ ATCC 27294 (Mycobacterium tuberculosis) [12].

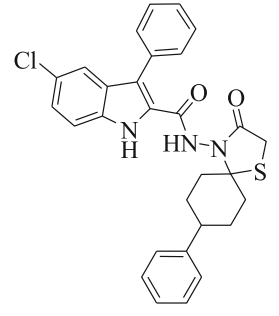

(154)

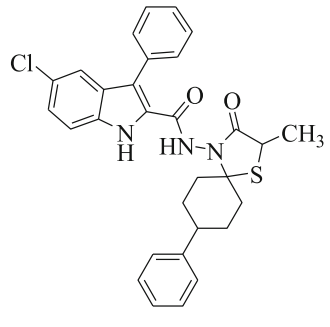

(155)
A library of indole chalcones was made by Ramesh et al. and screened for their antimycobacterial activity opposite $\mathrm{H}_{37} \mathrm{Rv}$ strain. Compounds (E)-1-(furan-3-yl)-3$(1 H$-indol-3-yl)prop-2-en-1-one $(156)(\mathrm{MIC}=210 \mu \mathrm{M})$, (E)-3-(1H-indol-3-yl)-1-(thiophen-2-yl)prop-2-en-1one $(157)$ MIC $=197 \mu \mathrm{M})$ and $(E)-2-((1 H$-indol-2yl)methylene)cyclopentan-1-one (158) $(\mathrm{MIC}=236 \mu \mathrm{M})$ were found to be potent drug against Mycobacterium tuberculosis [74].<smiles>O=C(/C=C/c1c[nH]c2ccccc12)c1ccccc1</smiles>

(156)

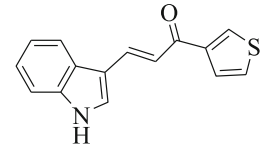

(157)

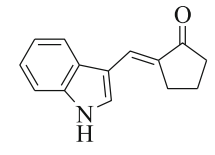

(158)
Naidu et al. synthesized various 3-(4-((1-(4-bromo3substitutedphenyl)-1H-1, 2, 3-triazol-4-yl) methyl) piperazin-1-yl) benzo[d]isoxazole derivatives as antitubercular agents against $\mathrm{H}_{37} \mathrm{Rv}$ strain Mycobacterium tuberculosis. Among the tested compound, compound 3-(4-((1-(4-bromo-3-(trifluoromethyl)phenyl)-1H-1,2,3-triazol-4-yl)methyl)piperazin-1-yl)benzo[d] isoxazole $(159)(\mathrm{MIC}=6.16 \mu \mathrm{M})$ exhibited best antitubercular activity. Receptor interactions were studied by docking to pantothenate synthetase enzyme [75].

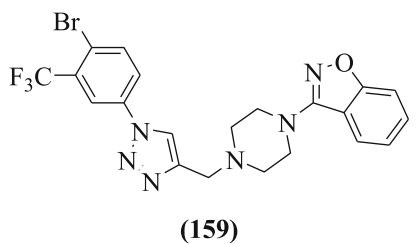

\section{Anticholinesterase activity}

Bingul et al. synthesized a novel 4, 6dimethoxyindole-based hydrazide hydrazones and evaluated their anticholinesterase activity towards cholinesterase (AChE and BuChE) enzymes. Compound methyl (E)-7-((2-benzoylhydrazono)methyl)-4, 6-dimethoxy- $1 H$-indole-2-carboxylate (160) was most active [76].<smiles>COC(=O)c1cc2c(OC)cc(OC)c(/C=N/NC(=O)c3ccccc3)c2[nH]1</smiles>

(160)

Prochnow et al. prepared 2-substituted- $\mathrm{N}$-alkynylindoles and screened for anticholinesterase activity. Derivatives (2-fluoro-6-methoxyphenyl)(1-(2-phenylethynyl)-1H-indol-2-yl)methanol (161) and (1-(2phenylethynyl)-1H-indol-2-yl)methanol (162) were 
found as potential inhibitors of cholinesterase activity [77].

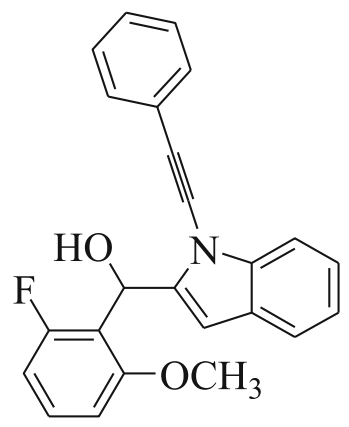

(161)

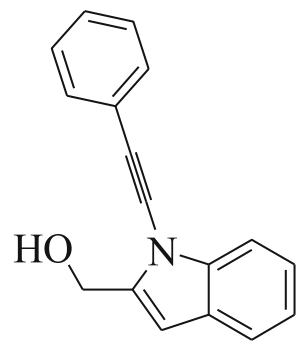

(162)
2-(2-(4-(Substitutedbenzyl) piperazin-1-yl) ethyl) isoindoline-1, 3-dione derivatives were reported as anticholinesterase agents by Mohammadi-Faran et al. Among synthesized derivatives, 2-(2-(4-(2-chlorobenzyl) piperazin-1-yl) ethyl) isoindoline-1, 3-dione (163) $\left(\mathrm{IC}_{50}=0.91 \pm 0.045 \mu \mathrm{M}\right)$ exhibited the highest activity with respect to the standard drug $\left(\mathrm{IC}_{50}=0.14 \pm\right.$ $0.03 \mu \mathrm{M})$ [78].<smiles>O=C1c2ccccc2C(=O)N1CCN1CCN(Cc2ccccc2Cl)CC1</smiles>

(163)

A novel 4, 6-dimethoxyindole-7-thiosemicarbazone derivatives were designed through Schiff base condensation reaction of indole carbaldehydes and thiosemicarbazides and evaluated for anticholinesterase properties by Bingül et al. Compound methyl (E)-4,6-dimethoxy-7-((2-(methylcarbamothioyl)hydrazono)methyl)- $1 H$-indole-2-carboxylate (164) exhibited moderate inhibition towards acetylcholinesterase and butyrylcholinesterase enzyme [79].<smiles>CNC(=S)N/N=C/c1c(OC)cc(OC)c2cc(C(=O)OC)[nH]c12</smiles>

(164)

1-(2-(4-(Substitutedbenzyl)piperazin-1-yl)acetyl)indoline-2,3-dione derivatives using piperazine and fluorobenzyl substituents were reported as acetylcholinesterase inhibitor by Ismail et al. Compound 1-(2-(4-(2fluorobenzyl)piperazin-1-yl)acetyl)indoline-2,3-dione (165) was found to exhibit the most active compound [80].<smiles>O=C1Cc2ccccc2N1C(=O)CN1CCN(Cc2ccccc2F)CC1</smiles>

(165)

Isoindoline-1, 3-dione derivatives were prepared as an inhibitor of acetylcholinesterase using Ellman's test by Aliabadi et al. Compound 2-(2-(4-(2-(4-Fluorophenyl)-2oxoethyl) piperazin-1-yl) ethyl) isoindoline-1, 3-dione (166) $\left(\mathrm{IC}_{50}=16.42 \pm 1.07 \mu \mathrm{M}\right)$ was active [81].<smiles>O=C(CN1CCN(CCN2C(=O)c3ccccc3C2=O)CC1)c1ccc(F)cc1</smiles>

(166)

Various urea and carbamates derived indole derivatives were reported for the inhibition of human monoamine oxidase-A (hMAO-A), human monoamine oxidase-B (hMAO-B), acetylcholinesterase (AChE), and butyrylcholinesterase (BuChE) by Denya et al. Molecular modeling showed significant interactions on active site of the enzyme. Compound $N, N$-Diethyl- $N^{\prime}$-[1-(prop-2-yn-1yl)- $1 H$-indol-6-yl] urea (167) was most potent [82].<smiles>C#CCN1C=CC2=CC=CN(C(=O)N(CC)CC)C21</smiles>

(167)

\section{Antimalarial activity}

Indole-3-glyoxyl tyrosine derivatives were reported by Vasconcelos et al. as antimalarial agents against the pathogen Plasmodium falciparum. Compounds (S)-methyl 2-(2-(1H-indol-3-yl)-2-oxoacetamido)-3(4'-fluoro-6-hydroxy-[1, 1'-biphenyl]-3-yl)propenoate (168) and $(S)$-methyl 2-(2-(1H-indol-3-yl)-2-oxoacetamido)-3-(4' -bromo-6-hydroxy-[1,1' -biphenyl]-3yl)propenoate (169) were favorable to antimalarial activity [83]. 
<smiles>COC(=O)C(Cc1cc(O)cc(-c2ccc(F)cc2)c1)NC(=O)C(=O)c1c[nH]c2ccccc12</smiles>

(168)

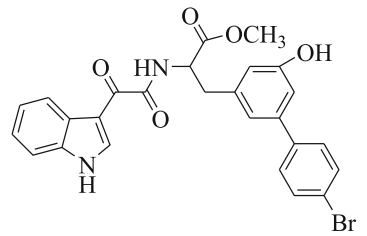

(169)
The potent antimalarial compounds were reported by Luthra et al. and compound $(Z)$-methyl 2-(2-((methylimino) (phenyl) methyl)- $1 H$-indol-3-yl) ethylcarbamate (170) was active at the trophozoite stage of the parasite growth [84].

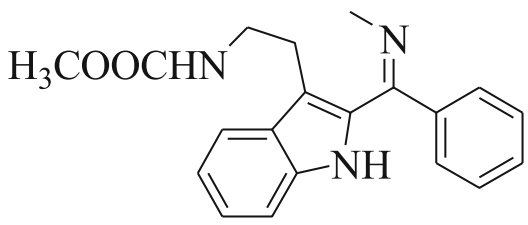

(170)

Schuck et al. synthesized melatonin compounds and assayed in Plasmodium falciparum culture for the measurement of antimalarial activities. Structural-activity relationship (SAR) showed that carboxamide group derivatives of indole gave a good result. Derivatives $\mathrm{N}$ (2-(5-methoxy- $1 \mathrm{H}$-indol-3-yl)ethyl)butyramide (171), $\mathrm{N}$ (2-(5-methoxy-1H-indol-3-yl)ethyl)hexanamide (172), and $\mathrm{N}$-(2-(5-methoxy-1H-indol-3-yl)ethyl)-2-phenylacetamide (173) were active at low concentration against the Plasmodium falciparum [85].<smiles>CCCC(=O)NCCc1c[nH]c2ccc(OC)cc12</smiles>

(171)

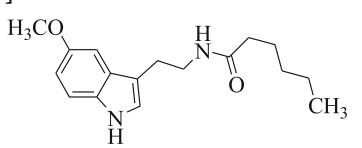

(172)

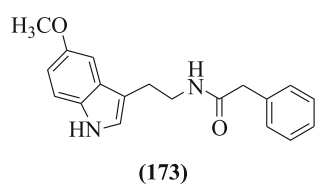

2-(1H-indol-3-yl)-4, 6-diphenylnicotinonitrile derivatives containing pyridine were made by Elshemy et al. and screened for antimalarial activity against Plasmodium falciparum. Among all the tested compounds, compounds 4-(4-fluorophenyl)-2-( $1 H$-indol-3-yl)-6-phenylnicotinonitrile (174), 4-(3,4-difluorocyclohexa-1,5-dienyl)-2-(1H-indol-3-yl)-6-phenylnicotinonitrile (175), and 2-(3H-inden-1-yl)-6-phenyl-4-(3,4,5-trimethoxycyclohexa-1,5-dienyl)nicotinonitrile (176) exhibit the highest selectivity index (S.I. ranged 3.8-10). Docking studies explained the interaction between compound and active site of the enzyme (quadruple mutant Plasmodium falciparum dihydrofolate reductase) [86]

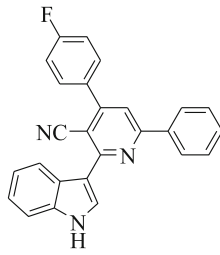

(174)

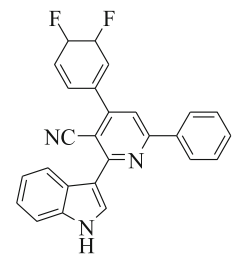

(175)

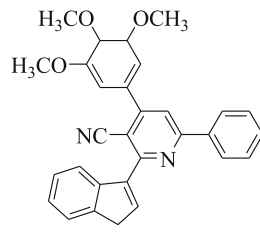

(176)

\section{Conclusion}

Indole moiety is present in many compounds possessing various biomedical applications. Various synthetic drug molecules contain an indole nucleus as a part of their pharmacophore structure and it helps in affixing drugs to the residues of the binding site of desired targets.

Derivatives holding indole core exhibit different biological activities namely antidiabetic, anticancer, antimicrobial, anti-HIV, antiviral, anti-inflammatory, antioxidant, anticholinesterase, antitubercular, and antimalarial activities, etc. Due to these activities, indole has attracted the attention of researchers in the discovery of novel chemical entities. These chemical entities may be safer and effective drugs for various ailments. Summarizing the literature reports described above, we can say that indole displays a diverse spectrum of biological activities. Indole has an immense potential to be investigated for newer therapeutic possibilities. Chemistry of indole derivatives described in this review would help the researchers worldwide in the design and synthesis of novel drugs useful in the mitigation of various disorders.

\section{Abbreviations}

LSD: Lysergic acid diethylamide; YFV: Yellow fever virus; BVDV: Bovine viral diarrhea virus; HIV-1: Human immunodeficiency virus; RSV: Respiratory syncytial virus; COX: Cyclooxygenase; FRAP: Ferric reducing ability of plasma; DPPH: 2,2-Diphenyl-1-picrylhydrazyl; ABTS: 2,2"-Azino-bis(3ethylbenzothiazoline-6-sulfonic acid; viz:: Specifically or namely; SAR: Structural activity relationship; $I C_{50}: I C_{50}$ is the concentration required to kill 50\% of cell population; hMAO-A: Human monoamine oxidase-A; hMAOB: Human monoamine oxidase-B; AChE: Acetylcholinesterase;

BuChE: Butyrylcholinesterase; MIC: Minimum inhibitory concentration; 'YM: Micromole; SI: Selectivity index; RNS: Reactive nitrogen species; ROS: Reactive oxygen species; MLT: Melatonin; DNA: Deoxyribonucleic acid; RNA: Ribonucleic acid; IFD: Induced-fit docking; HIV: Human

immunodeficiency viruses; NNRTIs: Non-nucleoside reverse transcriptase inhibitors; HBV: Hepatitis B virus; QSAR: Quantitative structure-activity relationship; TI: Therapeutic index; PI: Propidium iodide; CVS: Cardiovascular system; CNS: Central nervous system; GIT: Gastrointestinal tract; HMPH: 1-[(1H-indol-3yl)methylene]-2-phenylhydrazine; HSV-1: Herpes simplex type-1; sPLA 2 inhibitory: Secretary phospholipase $A_{2}$ inhibitory

\section{Acknowledgements}

The authors are thankful to the Department of Pharmaceutical Sciences, Guru Jambheshwar University of Science \& Technology, Hisar, Haryana, India for providing necessary facilities.

\section{Authors' contributions}

$\mathrm{R}$ collected the material and prepared the first draft of the manuscript. SK conceptualized, designed, and analyzed the work and prepared the final version for submission. All authors read and approved the final manuscript. 


\section{Funding}

No funding was available for this work.

\section{Availability of data and materials}

Not applicable.

Ethics approval and consent to participate

Not applicable.

\section{Consent for publication}

Not applicable.

\section{Competing interests}

The authors declare that they have no competing interests.

Received: 27 August 2020 Accepted: 5 November 2020

Published online: 12 December 2020

\section{References}

1. Lakhdar S, Westermaier M, Terrier F, Goumont R, Boubaker T, Ofial AR, Mayr H (2006) Nucleophilic reactivities of indoles. J Org Chem 71:9088+9095

2. Sharma V, Pradeep K, Devender P (2010) Biological importance of the indole nucleus in recent years: a comprehensive review. J Heterocycl Chem 47:491+502

3. Kaushik NK, Kaushik N, Attri P, Kumar N, Kim CH, Verma AK, Choi EH (2013) Biomedical Importance of Indoles. Molecules 18:6620+6662

4. Xue S, Ma L, Gao R, Lin Y, Linn Z (2014) Synthesis and antiviral activity of some novel indole-2-carboxylate derivatives. Acta Pharmaceutica Sinica B 4(4):313+321

5. Cihan-ì st ${ }^{1}$ ndag $G, G^{1}$ rsoy E, Naesens L, Ulusoy-G zeldemirci N, Ûapan G (2016) Synthesis and antiviral properties of novel indole-based thiosemicarbazides and 4-thiazolidinones. Bioorg Med Chem 24:240†246

6. Sellitto G, Faruolo A, Caprariis PD, Altamura S, Paonessa G, Ciliberto G (2010) Synthesis and anti-hepatitis $\mathrm{C}$ virus activity of novel ethyl $1 \mathrm{H}$-indole-3carboxylates in vitro. Bioorg Med Chem 18:6143+6148

7. Giampieri M, Balbia A, Mazzeia M, Collab PL, Ibba C, Loddo R (2009) Antiviral activity of indole derivatives. Antiviral Res 83:179+185

8. Tichy M, Pohl R, Xu HY, Chen YL, Yokokawa F, Shi PY, Hocek M (2012) Synthesis and antiviral activity of 4, 6-disubstituted pyrimido[4,5-b]indole ribonucleosides. Bioorgan Med Chem 20:6123+6133

9. Terzioglu N, Karali N, Gursoy A, Pannecouque C, Leysen P, Paeshuyse J, Neyts J, De Clercq E (2006) Synthesis and primary antiviral activity evaluation of 3-hydrazono-5-nitro-2-indolinone derivatives. Arkivoc 1:109† 118

10. El-Sawy AER, Abo-Salem HM, Zarie ES, Abd-Alla HI, El-Safty MM, Mandour $\mathrm{AH}$ (2015) Synthesis and antiviral activity of novel ethyl 2-(3-heterocycle- $1 \mathrm{H}$ indol-1-y/) acetate derivatives. Int J Pharm Pharm Sci 7(5):76+83

11. Abdel-gawad H, Mohamed HA, Dawood KM, Badria FAR (2010) Synthesis and antiviral activity of new indole-based heterocycles. Chem Pharm Bull 58(11):1529+1531

12. Cihan-ì st ${ }^{1}$ ndaą $G$, Naesens L, L’atana D, Erksse-Genÿ G, Mataracœe-Kara E, Ûapan G (2019) Design, synthesis, antitubercular and antiviral properties of new spirocyclic indole derivatives. Monatsh Chem 150(8):1533†1544

13. Ozdemir A, Altœntop MD, Zitouni GT, Ûiftÿi GA, Ertorun I, Alatas O, Kaplancœklœ ZA (2015) Synthesis and evaluation of new indole-based chalcones as potential antiinflammatory agents. Eur J Med Chem 89:304t 309

14. Sarva S, Harinah JS, Sthanikam SP, Ethiraj S, Vaithiyalingam M, Cirandur SR (2016) Synthesis, antibacterial and anti-inflammatory activity of bis(indolyl)methanes. Chinese Chem Lett 27:16+20

15. Rani P, Srivastava VK, Kumar A (2004) Synthesis and anti-inflammatory activity of heterocyclic indole derivatives. Eur J Med Chem 39:449+452

16. Pedada SR, Yarla NS, Tambade PJ, Dhananjaya BL, Bishayee A, Arunasree KM, Philip GH, Dharmapuri G, Aliev G, Putta S, Rangaiah G (2016) Synthesis of new secretory phospholipase A2-inhibitory indole containing isoxazole derivatives as anti-inflammatory and anticancer agents. Eur J Med Chem $112: 289+297$

17. Misra CS, Honnappa CG, Jitta SR, Gourishetti K, Daram P, Singh MP, Shrungeswara AH, Nayak Y, Unnikrishnan MK (2016) Biological activity of a small molecule indole analog, 1-[(1H-indol-3-y/)methylene]-2- phenylhydrazine $(\mathrm{HMPH})$, in chronic inflammation. Chem Biol Interact 244 $71+83$

18. Singh N, Bhati SK, Kumar A (2008) Thiazolyl /oxazolyl formazanyl indoles as potent anti-inflammatory agents. Eur J Med Chem 43:2597+2609

19. Amin NH, El-Saadi MT, Hefny AA, Abdelazeem AH, Elshemy HA, Abdellatif KR (2018) Anti-inflammatory indomethacin analogs endowed with preferential COX-2 inhibitory activity. Future Med Chem 10(21):2521+2535

20. Abdellatif KR, Lami PF, Omar HA (2016) 3-Methyl-2-phenyl-1-substitutedindole derivatives as indomethacin analogs: design, synthesis and biological evaluation as potential anti-inflammatory and analgesic agents. J Enzyme Inhib Med Chem 31(2):318+324

21. Chavan RS, More HN, Bhosale AV (2011) Synthesis, characterization and evaluation of analgesic and anti-inflammatory activities of some novel indoles. Trop J Pharmaceutical Res 10(4):463†473

22. Prajapati TR, Pandey DP, Gupta V, Joshi B, Dhingra GK (2018) Synthesis and anti-inflammatory activity of some newer potential isoxazoline derivatives of indole. Essence Int J Env Rehab Conserv IX(1):87+93

23. Mandour A, El-Sawy E, Shaker K, Mustafa M (2010) Synthesis, antiinflammatory, analgesic and anticonvulsant activities of 1, 8-dihydro-1-ary18-alkyl pyrazolo (3, 4-b) indoles. Acta Pharmaceutica 60(1):73+88

24. Bhat MA, Al-Omar MA, Raish M, Ansari MA, Abuelizz HA, Bakheit AH, Naglah AM (2018) Indole derivatives as cyclooxygenase inhibitors: synthesis, biological evaluation and docking studies. Molecules 23(6):1250

25. Ugwu DI, Okoro UC, Ukoha PO, Gupta A, Okafor SN (2018) Novel antiinflammatory and analgesic agents: synthesis, molecular docking and invivo studies. J Enzyme Inhib Med Chem 33(1):405†415

26. Shaker AM, Abdelall EK, Abdellatif KR, Abdel-Rahman HM (2018) Design, synthesis and biological evaluation of 2-(4-(methylsulfonyl) phenyl) indole derivatives with promising COX-2 inhibitory activity. J Appl Pharm Sci 8(11): $001+008$

27. Zhuang SH, Lin YC, Chou LC, Hsu MH, Lin HY, Huang CH, Lien JC, Kuo SC, Huang LJ (2013) Synthesis and anticancer activi ty of 2, 4-disubstituted furo[3,2-b]indole derivatives. Eur J Med Chem 66:466+479

28. Ma J, Bao G, Wang L, Li W, Xu B, Du B, Lv J, Zhai X, Gong P (2015) Design, synthesis, biologicalevaluation and preliminary mechanism study of novel benzothiazole derivatives bearing indole-based moiety as potent antitumor agents. Eur J Med Chem 96:173†186

29. Gurkan-Alp AS, Mumcuoglu M, Andac CA, Dayanc E, Cetin-Atalay R, Buyukbingol E (2012) Synthesis, anticancer activities and molecular modeling studies of novel indoleretinoid derivatives. Eur J Med Chem 58: $346+354$

30. Kumar TOS, Mahadavan KM, Kumara MN (2014) Synthesis and cytotoxic studies of 2, 3-dimethylindoles and tetrahydrocarbazoles. Int J Pharm Pharmaceutical Sci 6(2):2014

31. Han K, Wang H, Song B, Li Y, Ding WN, Zhao H, Sun H, Teng Y, Yu P (2014) Design, synthesis and biological activity evaluation of novel anticancer agent 5-(2-carboxyethenyl)indole derivatives. J Chem Pharm Res. 6(9):376† 380

32. Akkoc MK, Y Y Uksel MY, Durmaz I, Atalay RC (2012) Design, synthesis, and biological evaluation of indole-based 1, 4-disubstituted piperazines as cytotoxic agents. Turk J Chem 36:515†525

33. Kumar D, Kumar NM, Chang KH, Gupta R, Shah K (2011) Synthesis and invitro anticancer activity of 3, 5-bis (indolyl)-1, 2, 4-thiadiazoles. Bioorg Med Chem Lett 21:5897+5900

34. Choppara P, Prasad YV, Rao CV, Krishna KH, Trimoorthulu G, Rao GUM, Rao JV, Bethu MS, Murthy YLN (2015) Design, synthesis of novel N- prenylated indole-3-carbazones and evaluation of invitro cytotoxicity and 5-LOX inhibition activities. Arab J Chem xxx:xxx

35. Kaur H, Singh J, Narasimhan B (2019) Indole hybridized diazenyl derivatives: synthesis, antimicrobial activity, cytotoxicity evaluation and docking studies. BMC Chem 13(1):65

36. Yousif MN, Hussein HA, Yousif NM, El-Manawaty MA, El-Sayed WA (2019) Synthesis and anticancer activity of novel 2-phenylindole linked imidazolothiazole, thiazolo-s-triazine and imidazolyl-sugar systems. J Appl Pharm Sci 9(01):006+014

37. Kasralikar HM, Jadhavar SC, Bhusare SR (2015) Synthesis and molecular docking studies of oxochromenyl xanthenone and indolyl xanthenone derivatives as anti-HIV-1 RT inhibitors. Bioorg Med Chem Lett 25:3882†3886

38. Ran JQ, Huang N, Xu H, Yang LM, LV M, Zheng YT (2010) Anti HIV-1 agents 5: Synthesis and anti-HIV-1 activity of some $\mathrm{N}$-arylsulfonyl-3-acetylindoles invitro. Bioorg Med Chem Lett 20:3534†3536 
39. Selvam $P$, Chandramohan M, Clercq ED, Witvrouw M, Pannecouque C (2001) Synthesis and anti-HIV activity of 4-[(1, 2-dihydro-2-oxo-3H-indol-3ylidene) amino]-N (4, 6-dimethyl-2-pyrimidinyl)-benzene sulfonamide and its derivatives. Eur J Pharm Sci 14:313+316

40. Che Z, Tian Y, Liu S, Hu M, Chen G (2018) Discovery of N-arylsulfonyl-3acylindole benzoyl hydrazone derivatives as anti-HIV-1 agents. Brazilian J Pharmaceutical Sci 54(3)e17044:1-7

41. Ashok P, Lu CL, Chander S, Zheng YT, Murugesan S (2015) Design, synthesis, and biological evaluation of 1-(thiophen-2-yl)-9H-pyrido $[3,4-b]$ indole derivatives as anti-HIV-1 agents. Chem Biol Drug Des 85(6):722†728

42. Han X, Wu H, Wang W, Dong C, Tien P, Wu S, Zhou HB (2014) Synthesis and SARs of indole-based $Y$-amino acids as potent HIV-1 non-nucleoside reverse transcriptase inhibitors. Org Biomol Chem 12(41):8308+8317

43. Sanna G, Madeddu S, Giliberti G, Piras S, Struga M, Wrzosek M, KubiakTomaszewska G, Koziol AE, Savchenko O, Lis T, Stefanska J (2018) Synthesis and biological evaluation of novel indole-derived thioureas. Molecules 23(10):2554

44. Ragno R, Coluccia A, La Regina G, De Martino G, Piscitelli F, Lavecchia A, Novellino E, Bergamini A, Ciaprini C, Sinistro A, Maga G (2006) Design, molecular modeling, synthesis, and anti-HIV-1 activity of new indolyl aryl sulfones. Novel derivatives of the indole-2-carboxamide. J Med Chem 49(11):3172+3184

45. Chai $H$, Zhao $Y$, Zhao C, Gong P (2006) Synthesis and invitro anti-hepatitis B virus activities of some ethyl 6-bromo-5-hydroxy-1H-indole-3-carboxylates. Bioorgan Med Chem 14:911+917

46. Zhao C, Zhao Y, ChaiH GP (2006) Synthesis and in vitro anti-hepatitis B virus activities of some ethyl 5-hydroxy-1H-indole-3-carboxylates. Bioorgan Med Chem 14:2552+2558

47. Jiang T, Kuhen KL, Wolff K, Yin H, Bieza K, Caldwell J, Bursulaya B, Tuntland T, Zhang K, Karanewsky D, He Y (2006) Design, synthesis, and biological evaluations of novel oxindoles as HIV-1 non-nucleoside reverse transcriptase inhibitors. Part 2. Bioorg Med Chem Lett 16:2109+2112

48. Zhao Z, Wolkenberg SE, Lu M, Munshi V, Moyer G, Feng M, Carella AV, Ecto LT LT, Gabryelski L, Ming-Tain Lai MT, Prasad SG, Yan Y, McGaughey GB, Miller MD, Lindsley CW, Hartman GD, Vacca JP, Williams TM (2008) Novel indole-3-sulfonamides as potent HIV non-nucleoside reverse transcriptase inhibitors (NNRTIs). Bioorg Med Chem Lett 18:554†559

49. Chiummiento L, Funicello M, Lupattelli P, Tramutola F, Campaner P (2009) New indolic non-peptidic HIV protease inhibitors from (S)-glycidol: synthesis and preliminary biological activity. Tetrahedron 65:5984†5989

50. Ferro S, Luca LD, Barreca ML, Grazia SD, Christ F, Zeger Debyser Z, Chimirri A (2010) New chloro, fluorobenzylindole derivatives as integrase strandtransfer inhibitors (INSTIs) and their mode of action. Bioorgan Med Chem 18:5510+5518

51. Silveira CC, Mendes SR, Soares JR, Victoria FN, Martinez DM, Savegnago L (2013) Synthesis and antioxidant activity of new C-3 sulfenyl indoles. Tetrahedron Lett 54:4926+4929

52. Gurer-Orhan H, Karaaslan C, Ozcan S, Firuzi O, Tavakkoli M, Saso L, Suzen S (2016) Novel indole-based melatonin analogues: Evaluation of antioxidant activity and protective effect against amyloid $b$-induced damage. Bioorg Med Chem 24:1658†1664

53. Biradar JS, Sasidhar BS, Parveen R (2010) Synthesis, antioxidant and DNA cleavage activities of novel indole derivatives. Eur J Med Chem 45:4074†4078

54. Estevao MS, Carvalho LC, Ribeiro D, Couto D, Freitas M, Gomes A, Ferreira LM, Fernandes B, Marques MMB (2010) Antioxidant activity of unexplored indole derivatives: Synthesis and screening. Eur J Med Chem 45:4869†4878

55. Bakherad Z, Safavi M, Fassihi A, Sadeghi-Aliabadi H, Bakherad M, Rastegar H, Mahdavi M (2019) Anti-cancer, anti-oxidant and molecular docking studies of thiosemicarbazone indole-based derivatives. Res Chemical Intermediate 45(5): $2827+2854$

56. Suzen $\mathrm{S}$, Tekiner-Gulbas B, Shirinzadeh H, Uslu D, Gurer-Orhan H, Gumustas M, Ozkan SA (2013) Antioxidant activity of indole-based melatonin analogues in erythrocytes and their voltammetric characterization. J Enzyme Inhib Med Chem 28(6):1143†1155

57. Yœlmaz AD, Coban T, Suzen S (2012) Synthesis and antioxidant activity evaluations of melatonin-based analogue indole-hydrazide/hydrazone derivatives. J Enzyme Inhib Med Chem 27(3):428+436

58. Ummadi N, Gundala S, Venkatapuram P, Adivireddy P (2017) Synthesis and antioxidant activity of a new class of pyrazolyl indoles, thiazolyl pyrazolyl indoles. Med Chem Res 26(7):1574†1584
59. Ates-Alagoz Z, Coban T, Buyukbingol E (2006) Synthesis and antioxidant activity of new tetrahydro-naphthalene-indole derivatives as retinoid and melatonin analogs. Arch Pharm 339(4):193+200

60. Dixit A, Pathak D, Sharma GK (2020) Synthesis, antibacterial and free radical scavenging activity of some newer $\mathrm{N}-((10-$-nitro- $1 \mathrm{H}$-indolo $[1,2-c]$ quinazolin12-yl) methylene) benzenamines. Eur Pharmaceutical J 1 (ahead-of-print)

61. Xu H, Fan LL (2011) Antifungal agents. Part 4: Synthesis and antifungal activities of novel indole[1,2-c]-1,2,4-benzotriazine derivatives against phytopathogenic fungi invitro. Eur J Med Chem 46:364†369

62. Shi Z, Zhao Z, Huang M, Fu X (2015) Ultrasound-assisted, one-pot, threecomponent synthesis and antibacterial activities of novel indole derivatives containing 1,3,4-oxadiazole and 1,2,4-triazole moieties. C R Chimie 18:1320† 1327

63. Tiwari RK, Singh D, Singh J, Yadav V, Pathak AK, Dabur R, Chhillar AK, Singh R, Sharma GL, Chandra R, Verma AK (2006) Synthesis and antibacterial activity of substituted1, 2, 3, 4-tetrahydropyrazino [1, 2-a] indoles. Bioorg Med Chem Lett 16:413+416

64. Hong W, Li J, Chang Z, Tan X, Yang H, Ouyang Y, Yang Y, Kaur S, Paterson IC, Ngeow YF, Wang H (2017) Synthesis and biological evaluation of indole core-based derivatives with potent antibacterial activity against resistant bacterial pathogens. J Antibiotics 70:832+844

65. Ozturk A, Abdullah M (2006) Toxicological effect of indole and its azo dye derivatives on some microorganisms under aerobic conditions. Sci Total Environ 358:137†142

66. Scribner A, Moore JA III, Ouvry G, Fisher M, Wyvratt M, Leavitt $P$, Liberator $P$, Gurnett A, Brown C, Mathew J, Thompson D, Schmatz D, Biftu T (2009) Synthesis and biological activity of anticoccidial agents: 2,3-diarylindoles. Bioorg Med Chem Lett 19:1517†1521

67. Singh P, Verma P, Yadav B, Komath SS (2011) Synthesis and evaluation of indole-based new scaffolds for antimicrobial activities and Identification of promising candidates. Bioorg Med Chem Lett 21:3367†3372

68. Desai NC, Somani H, Trivedi A, Bhatt K, Nawale L, Khedkar VM, Jha PC, Sarkar D (2016) Synthesis, biological evaluation and molecular docking study of some novel indole and pyridine based 1, 3, 4-oxadiazole derivatives as potential antitubercular agents. Bioorg Med Chem Lett 26:1776†1783

69. Guo S, Tipparaju SK, Pegan SD, Wanc B, Moa S, Orjala J, Mesecar AD AD, Franzblau SG, Kozikowski AP (2009) Natural product leads for drug discovery: Isolation, synthesis and biological evaluation of 6-cyano-5methoxyindolo[2,3-a]carbazole based ligands as antibacterial agents. Bioorgan Med Chem 17:7126+7130

70. Velezheva V, Brennan P, Ivanov P, Kornienko A, Lyubimov S, Kazarian K, Nikonenko B, Majorov K, Apt A (2016) Synthesis and antituberculosis activity of indoletpyridine derived hydrazides, hydrazidethydrazones, and thiosemicarbazones. Bioorg Med Chem Lett 26(3):978†985

71. Walmik P, Saundane AR (2014) Synthesis of novel indolyl-azetidinone and thiazolidinone derivatives as a potent antioxidant, antimicrobial and antitubercular agents. Der Pharma Chemica 6(4):70+79

72. Rathod AS, Godipurge SS, Biradar JS (2017) Synthesis of indole, coumarinyl and pyridinyl derivatives of isoniazid as potent antitubercular and antimicrobial agents and their molecular docking studies. Int J Pharm Pharm Sci 9:233+240

73. Kondreddi RR, Jiricek J, Rao SP, Lakshminarayana SB, Camacho LR, Rao R, Herve M, Bifani P, Ma NL, Kuhen K, Goh A, Chatterjee AK, Dick T, Diagana TT, Manjunatha UN, Smith PW (2013) Design, synthesis, and biological evaluation of indole-2-carboxamides: a promising class of antituberculosis agents. J Med Chem 56(21):8849†8859

74. Ramesh D, Joji A, Vijayakumar BG, Sethumadhavan A, Mani M, Kannan T (2020) Indole chalcones: Design, synthesis, invitro and insilico evaluation against Mycobacterium tuberculosis. Eur J Med Chem 198:112358. https://doi. org/10.1016/j.ejmech.2020.112358

75. Naidu KM, Srinivasarao S, Agnieszka N, Ewa AK, Kumar MMK, Sekhar KVGC (2016) Seeking potent anti-tubercular agents: Design, synthesis, antitubercular activity and docking study of various ((triazoles/indole)-piperazin1-yl/1, 4-diazepan-1-yl) benzo [d] isoxazole derivatives. Bioorg Med Chem Lett 26(9):2245+2250

76. Bingul M, Ercan S, Boga M (2020) The design of novel 4, 6-dimethoxyindole based hydrazide-hydrazones: Molecular modeling, synthesis and anticholinesterase activity. J Mol Struct 1213:128202

77. Prochnow T, Maroneze A, Back DF, Jardim NS, Nogueira CW, Zeni G (2018) Synthesis and anticholinesterase activity of 2-substituted-N-alkynylindoles. Org Biomol Chem 16(42):7926+7934 
78. Mohammadi-Farani A, Ahmadi A, Nadri H, Aliabadi A (2013) Synthesis, docking and acetylcholinesterase inhibitory assessment of 2-(2-(4Benzylpiperazin-1-yl) ethyl) isoindoline-1, 3-dione derivatives with potential anti-Alzheimer effects. DARU J Pharma Sci 21(1):47

79. Bing ${ }^{1}$ I M (2019) Synthesis and characterisation of novel 4, 6dimethoxyindole-7-and-2-thiosemicarbazone derivatives: Biological evaluation as antioxidant and anticholinesterase candidates. J Chem Res 43(9-10):399+406

80. Ismail MM, Kamel MM, Mohamed LW, Faggal SI (2012) Synthesis of new indole derivatives structurally related to donepezil and their biological evaluation as acetylcholinesterase inhibitors. Molecules 17(5):4811†4823

81. Aliabadi A, Foroumadi A, Mohammadi-Farani A, Mahvar MG (2013) Synthesis and evaluation of anti-acetylcholinesterase activity of 2-(2-(4-(2-Oxo-2phenylethyl) piperazin-1-yl) ethyl) isoindoline-1, 3-dione derivatives with potential anti-Alzheimer effects. Iran J Basic Medl sci 16(10):1049

82. Denya I, Malan SF, Enogieru AB, Omoruyi SI, Ekpo OE, Kapp E, Zindo FT, Joubert J (2018) Design, synthesis and evaluation of indole derivatives as multifunctional agents against Alzheimer"s disease. Med Chem Comm 9(2): $357+370$

83. Vasconcelos SN, Meissner KA, Ferraz WR, Trossini GH, Wrenger C, Stefani HA (2019) Indole-3-glyoxyl tyrosine: synthesis and antimalarial activity against Plasmodium falciparum. Future Med Chem 11(6):525†538

84. Luthra T, Nayak AK, Bose S, Chakrabarti S, Gupta A, Sen S (2019) Indole based antimalarial compounds targeting the melatonin pathway: their design, synthesis and biological evaluation. Eur J Med Chem 168:11+27

85. Schuck DC, Jordùo AK, Nakabashi M, Cunha AC, Ferreira VF, Garcia CR (2014) Synthetic indole and melatonin derivatives exhibit antimalarial activity on the cell cycle of the human malaria parasite Plasmodium falciparum. Eur J Med Chem 78:375+382

86. Elshemy HA, Zaki MA, Mohamed El, Khan SI, Lamie PF (2020) A multicomponent reaction to design antimalarial pyridyl-indole derivatives: Synthesis, biological activities and molecular docking. Bioorg Chem 97: 103673

\section{Publisher $\div$ s Note}

Springer Nature remains neutral with regard to jurisdictional claims in published maps and institutional affiliations.

\section{Submit your manuscript to a SpringerOpen ${ }^{\circ}$ journal and benefit from:}

- Convenient online submission

- Rigorous peer review

- Open access: articles freely available online

- High visibility within the field

- Retaining the copyright to your article

Submit your next manuscript at $\boldsymbol{\nabla}$ springeropen.com 